\title{
Subexponential Time and Fixed-Parameter Tractability: Exploiting the Miniaturization Mapping
}

\author{
Yijia Chen * \\ Shanghai Jiaotong University
}

\author{
Jörg Flum ${ }^{\dagger}$ \\ Albert-Ludwigs-Universität Freiburg
}

August 20, 2008

\begin{abstract}
Recently it has been shown that the miniaturization mapping $\mathscr{M}$ faithfully translates subexponential parameterized complexity into (unbounded) parameterized complexity. We determine the preimages under $\mathscr{M}$ of various (classes of) problems. For many parameterized problems whose underlying classical problem is in NP we show that the preimages coincide with natural reparameterizations which take into account the amount of nondeterminism needed to solve them.
\end{abstract}

Keywords. Parameterized complexity, exponential time complexity, miniaturization mapping

\section{Introduction}

The idea of fixed-parameter tractability is to approach hard algorithmic problems by isolating problem parameters that can be expected to be small in certain applications and then develop algorithms that are polynomial except for an arbitrary dependence on the parameter. More precisely: A parameterized problem is a pair $(Q, \kappa)$, where $Q$ is a classical problem, say, over the alphabet $\Sigma$ and $\kappa: \Sigma^{*} \rightarrow \mathbb{N}$ is a polynomial time computable function assigning to every $x \in \Sigma^{*}$ its parameter $\kappa(x)$. The problem $(Q, \kappa)$ is fixed-parameter tractable if it can be solved by an algorithm the running time of which is bounded by $f(\kappa(x)) \cdot|x|^{O(1)}$, where $f$ is an arbitrary computable function. The class of all fixed-parameter tractable problems is denoted by FPT.

There are natural problems that are fixed-parameter tractable, but require a parameter dependence $f$ of the form $f(k):=2^{2^{2^{k}}}$ or even worse. However, even for small values of the parameter $\kappa(x)$, a running time such as $2^{2^{2^{\kappa(x)}}} \cdot|x|$ is prohibitive. Hence, besides the unbounded fixed-parameter tractability, more restrictive notions of tractability have been studied obtained by simply putting upper bounds on the growth of the "parameter dependence" $f$, the most restrictive ones considered so far being $f \in 2^{O(k)}$ and $f \in 2^{o(k)}$. ${ }^{1}$ The corresponding class of "tractable" problems have been denoted by EPT and SUBEPT, respectively, and the theory corresponding to SUBEPT by subexponential parameterized complexity.

It is a beautiful aspect of subexponential parameterized complexity theory that it can be faithfully translated into unbounded parameterized complexity theory via the miniaturization mapping $\mathscr{M}$ : Let $(Q, \kappa)$ be a parameterized problem over the alphabet $\Sigma$. The miniaturization $\mathscr{M}(Q, \kappa)$ of $(Q, \kappa)$ is the parameterized problem

\footnotetext{
*Email: yijia.chen@cs.sjtu.edu.cn

†Email: joerg.flumemath.uni-freiburg.de

${ }^{1}$ In the precise definition given in Section 2 we have to require $f \in 2^{e^{\text {eff }}(k)}$ (which is an effective version of $f \in 2^{o(k)}$ ); this stronger requirement is necessary in order to relate it to our (strongly uniform) fixed-parameter tractability.
} 


$$
\begin{aligned}
\mathscr{M}(Q, \kappa) & \\
\text { Instance: } & x \in \Sigma^{*} \text { and } m \in \mathbb{N} \text { in unary. } \\
\text { Parameter: } & {\left[\frac{\kappa(x)}{\log m}\right] . } \\
\text { Question: } & \text { Is } x \in Q ?
\end{aligned}
$$

As just remarked the miniaturization mapping $\mathscr{M}$ establishes a connection between the subexponential theory and the unbounded theory and thereby $\mathscr{M}$ maps SUBEPT onto FPT. The mapping $\mathscr{M}$ strongly preserves reducibilities and every parameterized problem in XP has a preimage under the miniaturization map in EPT. Here XP denotes the class of parameterized problems $(Q, \kappa)$ solvable in time $O\left(|x|^{f(\kappa(x))}\right)$ for some computable $f$. To state the precise result, proven in [3], we write $(Q, \kappa) \leq^{\text {serf }}\left(Q^{\prime}, \kappa^{\prime}\right)$ and $(Q, \kappa) \leq^{\text {fpt }}\left(Q^{\prime}, \kappa^{\prime}\right)$ if there are a serf-reduction and an fpt-reduction, respectively, from $(Q, \kappa)$ to $\left(Q^{\prime}, \kappa^{\prime}\right)$. Serf-reductions and fpt-reductions are the standard notions of many-one reductions in subexponential parameterized complexity and in unbounded parameterized complexity, respectively (compare Section 2 for the definitions).

Theorem 1 (Miniaturization Theorem). (1) Let $(Q, \kappa)$ be a parameterized problem. Then

$$
(Q, \kappa) \in \mathrm{SUBEPT} \Longleftrightarrow \mathscr{M}(Q, \kappa) \in \mathrm{FPT} .
$$

(2) Let $(Q, \kappa)$ and $\left(Q^{\prime}, \kappa^{\prime}\right)$ be parameterized problems. Then

$$
(Q, \kappa) \leq^{\operatorname{serf}}\left(Q^{\prime} \kappa^{\prime}\right) \Longleftrightarrow \mathscr{M}(Q, \kappa) \leq^{\mathrm{fpt}} \mathscr{M}\left(Q^{\prime}, \kappa^{\prime}\right) .
$$

(3) Let $(Q, \kappa) \in \mathrm{XP}$. Then there exists a problem $\left(Q^{\prime}, \kappa^{\prime}\right)$ in EPT such that

$$
\mathscr{M}\left(Q^{\prime}, \kappa^{\prime}\right) \equiv^{\mathrm{fpt}}(Q, \kappa) .
$$

The class XP comprises the most important hierarchy of classes of intractable parameterized problems, namely the W-hierarchy. We recall a property of this hierarchy. For a set $\Gamma$ of propositional formulas the parameterized weighted satisfiability problem for $\Gamma$ is defined by

$$
\begin{aligned}
p \text {-WS AT }(\Gamma) & \\
\text { Instance: } & \text { A propositional formula } \alpha \in \Gamma \text { and } k \in \mathbb{N} . \\
\text { Parameter: } & k . \\
\text { Question: } & \text { Does } \alpha \text { have a satisfying assignment of weight } k ?^{2}
\end{aligned}
$$

Then for the classes $\mathrm{W}[1], \mathrm{W}[2], \ldots$ of the $\mathrm{W}$-hierarchy the following holds:

- If $t, d \geq 1$ and $t+d \geq 3$, then $p$-WSAT $\left(\Gamma_{t, d}\right)$ is $\mathrm{W}[t]$-complete under fpt-reductions.

Here $\Gamma_{t, d}$ contains the propositional formulas that are big conjunctions of big disjunctions of big conjunctions ... ( $t$ alternations) of conjunctions (if $t$ is even) and disjunctions (if $t$ is odd) of $d$ literals (see Section 5 for the precise definition).

In [3] the authors consider the reparameterization form-WSAT $(\Gamma)$ of $p$ - $\operatorname{WSAT}(\Gamma)$, where

$$
\begin{aligned}
\text { form-WSAT }(\Gamma) & \\
\text { Instance: } & \text { A formula } \alpha \in \Gamma \text { and } k \geq 1 . \\
\text { Parameter: } & k \cdot\lceil\log |\alpha|\rceil . \\
\text { Question: } & \text { Does } \alpha \text { have a satisfying assignment of weight } k ?
\end{aligned}
$$

They show for the $\Gamma$ 's relevant for the W $[t]$ 's that the problem form-WSAT $(\Gamma)$ is the preimage of $p$-WSAT $(\Gamma)$ under the miniaturization mapping, that is:

\footnotetext{
${ }^{2}$ The weight of an assignment for the variables of $\alpha$ is the number of variables set to TRUE.
} 
- If $t, d \geq 1$ and $t+d \geq 3$, then $\mathscr{M}\left(\right.$ form-WSAT $\left.\left(\Gamma_{t, d}\right)\right) \equiv{ }^{\mathrm{fpt}} p-\mathrm{WSAT}\left(\Gamma_{t, d}\right)$.

Already an analysis of the proof of part (3) of the Miniaturization Theorem shows that the preimage of a parameterized problem $(Q, \kappa)$ under the miniaturization mapping essentially is a reparameterization of $(Q, \kappa)$. How do we get this reparameterization? Is there a natural reparameterization that is a preimage? Can we determine the preimages of a large class of problems? These are the problems we address in this paper. In [3] the authors prove that the reparameterization of problems obtained by multiplying the parameter by the logarithm of the size of the instance gives an inverse for the miniaturization mapping for all problems satisfying certain technical conditions. Essentially form-WSAT $(\Gamma)$ is this reparameterization of $p$-WSAT $(\Gamma)$. We generalize this approach here.

Let $(Q, \kappa)$ be a parameterized problem over the alphabet $\Sigma$. Often (the classical problem) $Q$ has a canonical representation of the form

$$
x \in Q \quad \Longleftrightarrow \quad \text { there is a } y \in\{0,1\}^{g(x)} \text { such that }(x, y) \in Q_{0}
$$

for some polynomial time computable function $g: \Sigma^{*} \rightarrow \mathbb{N}$ and some $Q_{0} \in$ PTIME. (We give such a representation for $Q:=$ WSAT $(\Gamma)$ below.) Consider the nondeterministic algorithm solving $x \in Q$ by guessing $y \in\{0,1\}^{g(x)}$ and then verifying that $(x, y) \in Q_{0}$ in polynomial time. The deterministic procedure simulating all possible computation paths of this nondeterministic algorithm takes time $2^{g(x)} \cdot|x|^{O(1)}$. Often the question arises whether we can do better and solve the problem $x \in Q$ in time

$$
2^{o(g(x))} \cdot|x|^{O(1)},
$$

which is equivalent to $(Q, g) \in \operatorname{SUBEPT}$. We call $(Q, g)$ the canonical parameterization of $Q$ (more precisely, one should speak of the canonical parameterization induced by the representation (1) of $Q$ ) and sometimes we say that $(Q, g)$ is the canonical reparameterization of $(Q, \kappa)$.

Let $Q$ be the weighted satisfiability problem $\operatorname{WSAT}(\Gamma)$ for formulas in $\Gamma$, that is, $(\alpha, k) \in$ $Q$ if and only if $\alpha \in \Gamma$ and $\alpha$ has a satisfying assignment of weight $k$. A canonical representation of $Q$ of the form (1) is

$$
(\alpha, k) \in Q \quad \Longleftrightarrow \quad \text { there is a } y \in\{0,1\}^{k \cdot[\log |\operatorname{var}(\alpha)|\rceil} \text { such that }((\alpha, k), y) \in Q_{0},
$$

where $\operatorname{var}(\alpha)$ is the set of variables of $\alpha$ and $((\alpha, k), y) \in Q_{0}$ means that $\alpha \in \Gamma$, that $y$ contains the binary representation of $k$ distinct variables of $\alpha$ and that the assignment setting exactly these variables to TRUE satisfies $\alpha$. Clearly $Q_{0}$ is in PTIME if $\Gamma$ is. Hence the canonical parameterization is the problem

$$
\begin{aligned}
\text { var-WSAT }(\Gamma) & \\
\text { Instance: } & \text { A formula } \alpha \in \Gamma \text { and } k \geq 1 . \\
\text { Parameter: } & \lceil k \cdot \log |\operatorname{var}(\alpha)|\rceil . \\
\text { Question: } & \text { Does } \alpha \text { have a satisfying assignment of weight } k ?
\end{aligned}
$$

We show that in addition to form- $\operatorname{WSAT}\left(\Gamma_{t, d}\right)$ also var-WSAT $\left(\Gamma_{t, d}\right)$ is a preimage of the problem $p$ - $\mathrm{WSAT}\left(\Gamma_{t, d}\right)$, that is:

$$
\text { - If } t, d \geq 1 \text { and } t+d \geq 3 \text {, then } \mathscr{M}\left(\operatorname{var}-\mathrm{WSAT}\left(\Gamma_{t, d}\right)\right) \equiv{ }^{\mathrm{fpt}} p-\operatorname{WSAT}\left(\Gamma_{t, d}\right) .
$$

The main question we address is: For what other parameterized problems is the canonical reparameterization a preimage under the miniaturization map?

In Section 3 we show that the answer is positive for the parameterized dominating set problem. In Section 4 we introduce a general framework that allows us to carry out the arguments we use for the dominating set problem. In Section 5 we apply this abstract approach to reprove and extend the results obtained in [3] for the weighted satisfiability problem. Perhaps the most far-reaching positive answer to our main question is obtained in Section 6: 
If $t \geq 1$ and the parameterized problem $(Q, \kappa)$ is $\mathrm{W}[t]$-complete and Fagindefinable by a $\Pi_{t}$-formula, then the preimage of $(Q, \kappa)$ under the miniaturization mapping is its canonical reparameterization.

To broaden the range of applicability of this result we deal with relativized Fagin-definable problems. As an application we get:

If one can decide whether a hypergraph $\mathcal{H}$ has a hitting set of size $k$ in time $2^{o(k \cdot \log (|V|+|E|))} \cdot\|\mathcal{H}\|^{O(1)}$, then one can even decide it in time $2^{o(k \cdot \log |V|)}$. $\|\mathcal{H}\|^{O(1)}$.

In Section 7 we discuss the main question for model-checking problems and give an application to the homomorphism problem. Finally, in Section 8 we determine parameterized problems complete in the preimages of the classes of the A-hierarchy. Here we face the additional difficulty that problems complete in some class of the A-hierarchy have an underlying classical problem which is not in NP (unless A[2] $\subseteq$ para-NP), so that we have no notion of canonical reparameterization.

As in [8] we consider the miniaturization mapping as an isomorphism between subexponential parameterized complexity and unbounded parameterized complexity. The authors of [3] view the mapping as an isomorphism between exponential complexity and unbounded parameterized complexity. On the side of the exponential theory the parameterization is viewed as a size function. We strongly recommend the worth reading introduction of [3].

\section{Some basic notions from Parameterized Complexity}

The set of natural numbers (that is, nonnegative integers) is denoted by $\mathbb{N}$. For a natural number $n$ let $[n]:=\{1, \ldots, n\}$. By $\log n$ we mean $\lceil\log n\rceil$ if an integer is expected. For $n=0$ the term $\log n$ is undefined. We trust the reader's common sense to interpret such terms reasonably.

Recall from the Introduction that a parameterized problem is a pair $(Q, \kappa)$, where $Q$ is a classical problem, say, over the alphabet $\Sigma$ and $\kappa: \Sigma^{*} \rightarrow \mathbb{N}$ is a polynomial time computable function assigning to every $x \in \Sigma^{*}$ its parameter $\kappa(x)$. We also recall the definition of FPT and SUBEPT, the classes of tractable problems from the point of view of (unbounded) fixedparameter tractability and of subexponential fixed-parameter tractability, respectively.

The problem $(Q, \kappa)$ is fixed-parameter tractable if there is an algorithm solving $x \in Q$ in time $f(\kappa(x)) \cdot|x|^{O(1)}$, where $f$ is a computable function. The class of all fixed-parameter tractable problems is denoted by FPT.

Let $f, g: \mathbb{N} \rightarrow \mathbb{N}$ be computable functions. Then $f \in o^{\text {eff }}(g)$ if there is a computable function $h$ such that for all $\ell \geq 1$ and $k \geq h(\ell)$,

$$
f(k) \leq \frac{g(k)}{\ell}
$$

or, equivalently, $f \in o^{\text {eff }}(g)$ if there exist $k_{0} \in \mathbb{N}$ and a computable function $\iota$ that is nondecreasing and unbounded such that for all $k \geq k_{0}$,

$$
f(k) \leq \frac{g(k)}{\iota(k)} .
$$

We often write $f(k) \in o^{\text {eff }}(g(k))$ instead of $f \in o^{\text {eff }}(g)$. A parameterized problem $(Q, \kappa)$ is in SUBEPT if $x \in Q$ is solvable in time $f(\kappa(x)) \cdot|x|^{O(1)}$ for some computable function $f$ with $f(k) \in 2^{o^{\text {eff }}(k)}$.

We recall the notion of reducibility for the unbounded and for the subexponential parameterized complexity. Let $(Q, \kappa)$ and $\left(Q^{\prime}, \kappa^{\prime}\right)$ be parameterized problems over the alphabets $\Sigma$ and $\Sigma^{\prime}$, respectively.

An fpt-reduction from $(Q, \kappa)$ to $\left(Q^{\prime}, \kappa^{\prime}\right)$ is a mapping $R: \Sigma^{*} \rightarrow\left(\Sigma^{\prime}\right)^{*}$ such that: 
- For all $x \in \Sigma^{*}$ we have

$$
x \in Q \Longleftrightarrow R(x) \in Q^{\prime} .
$$

- There is a computable $f: \mathbb{N} \rightarrow \mathbb{N}$ such that for all $x \in \Sigma^{*}$, the value $R(x)$ is computable in time

$$
f(\kappa(x)) \cdot|x|^{O(1)} .
$$

- There is a computable function $g: \mathbb{N} \rightarrow \mathbb{N}$ such that $\kappa^{\prime}(R(x)) \leq g(\kappa(x))$ for all $x \in \Sigma^{*}$.

We write $(Q, \kappa) \leq^{\mathrm{fpt}}\left(Q^{\prime}, \kappa^{\prime}\right)$ if there is an fpt-reduction from $(Q, \kappa)$ to $\left(Q^{\prime}, \kappa^{\prime}\right)$, and we write $(Q, \kappa) \equiv \equiv^{\mathrm{fpt}}\left(Q^{\prime}, \kappa^{\prime}\right)$ if $(Q, \kappa) \leq^{\mathrm{fpt}}\left(Q^{\prime}, \kappa^{\prime}\right)$ and $\left(Q^{\prime}, \kappa^{\prime}\right) \leq^{\mathrm{fpt}}(Q, \kappa)$.

The classes $\mathrm{W}[1], \mathrm{W}[2], \ldots$ of the W-hierarchy, the classes $\mathrm{W}[\mathrm{SAT}]$ and $\mathrm{W}[\mathrm{P}]$, and the classes A[1], A[2], .. of the A-hierarchy are defined in (or, characterized by) Theorem 13 and Proposition 36, respectively, via complete problems under fpt-reductions.

A subexponential reduction family, or simply serf-reduction, from $(Q, \kappa)$ to $\left(Q^{\prime}, \kappa^{\prime}\right)$ is a mapping $S: \Sigma^{*} \times \mathbb{N} \rightarrow\left(\Sigma^{\prime}\right)^{*}$ such that:

- For all $(x, \ell) \in \Sigma^{*} \times \mathbb{N}$ we have

$$
x \in Q \Longleftrightarrow S(x, \ell) \in Q^{\prime} .
$$

- There is a computable function $f$ such that for all $(x, \ell) \in \Sigma^{*} \times \mathbb{N}$ the value $S(x, \ell)$ is computable in time

$$
f(\ell) \cdot 2^{\kappa(x) / \ell} \cdot|x|^{O(1)} .
$$

- There is a computable function $g$ such that for all $(x, \ell) \in \Sigma^{*} \times \mathbb{N}$,

$$
\kappa^{\prime}(S(x, \ell)) \leq g(\ell) \cdot(\kappa(x)+\log |x|) .
$$

We write $(Q, \kappa) \leq^{\text {serf }}\left(Q^{\prime}, \kappa^{\prime}\right)$ if there is a serf-reduction from $(Q, \kappa)$ to $\left(Q^{\prime}, \kappa^{\prime}\right)$ and use the derived notation $\equiv^{\text {serf }}$.

Note that $(Q, \kappa) \leq^{\text {serf }}\left(Q^{\prime}, \kappa^{\prime}\right)$ if there is a mapping $R: \Sigma^{*} \rightarrow\left(\Sigma^{\prime}\right)^{*}$ such that:

- For all $x \in \Sigma^{*}$ we have $\left(x \in Q \Longleftrightarrow R(x) \in Q^{\prime}\right)$.

- $R(x)$ is computable in time $2^{o^{\text {eff }}(\kappa(x))} \cdot|x|^{O(1)}$.

$-\kappa^{\prime}(R(x) \leq O(\kappa(x))$.

As then $S(x, \ell):=R(x)$ is a serf-reduction, we often call $R$ a serf-reduction.

\section{An example}

We consider the parameterized dominating set problem

$$
\begin{aligned}
& p \text {-DominATING-SET } \\
& \text { Instance: } \text { A graph } \mathcal{G}=(V, E) \text { and } k \in \mathbb{N} . \\
& \text { Parameter: } k . \\
& \text { Question: } \text { Does } \mathcal{G} \text { have a dominating set of size } k \text { ? }
\end{aligned}
$$

and its canonical reparameterization

uni-DOMINATING-SET

Instance: $\quad$ A graph $\mathcal{G}=(V, E)$ and $r \in \mathbb{N}$.

Parameter: $\quad r \cdot \log |V|$.

Question: Does $\mathcal{G}$ have a dominating set of size $r$ ? 
and show (recall the definition of the miniaturization $\mathscr{M}(Q, \kappa)$ of $(Q, \kappa)$ from the Introduction):

Theorem 2. $\mathscr{M}($ uni-Dominating-Set $) \equiv{ }^{\mathrm{fpt}} p$-Dominating-Set.

Proof: $p$-Dominating-SeT $\leq \leq^{\mathrm{fpt}} \mathscr{M}$ (uni-DominATING-SeT): Let $(\mathcal{G}, k)$ be an instance of $p$-Dominating-SET with $\mathcal{G}=(V, E)$. We set

$$
r:=k \quad \text { and } \quad m:=|V| \text {. }
$$

The instance $(\mathcal{G}, r, m)$ of $\mathscr{M}$ (uni-DominatiNG-SET) has the parameter

$$
\left\lceil\frac{r \cdot \log |V|}{\log m}\right\rceil=k
$$

hence, the mapping $(\mathcal{G}, k) \mapsto(\mathcal{G}, r, m)$ is an fpt-reduction.

$\mathscr{M}($ uni-Dominating-SeT $) \leq{ }^{\mathrm{fpt}} p$-Dominating-SeT: Let $(\mathcal{G}, r, m)$ be an instance of the problem $\mathscr{M}($ uni-DominATING-SET) with $\mathcal{G}=(V, E)$. We can assume that

$$
2 \leq r \leq|V| .
$$

Recall that the parameter of $(\mathcal{G}, r, m)$ is

$$
k:=\left\lceil\frac{r \cdot \log |V|}{\log m}\right\rceil .
$$

Now we distinguish two cases. If $|V|>m$, then $k>r$ by (3). It follows that we can map $(\mathcal{G}, r, m)$ to the equivalent instance $(\mathcal{G}, r)$ of $p$-DOMINATING-SET (its parameter $r$ is bounded by $k$ ). In case $|V| \leq m$ we have

$$
k \leq r .
$$

Moreover, $r \cdot \log |V| \leq k \cdot \log m$ and hence

$$
|V|^{r / k} \leq m
$$

We want to get from the instance $(\mathcal{G}, r, m)$ of $\mathscr{M}$ (uni-DominATING-SET) in fpt-time a pair $\left(\mathcal{G}^{\prime}, k^{\prime}\right)$ such that

$$
\mathcal{G} \text { has a dominating set of size } r \quad \Longleftrightarrow \quad \mathcal{G}^{\prime} \text { has a dominating set of size } k^{\prime}
$$

and such that $k^{\prime} \leq g(k)$ for some computable $g$. The next lemma, which finishes this proof, shows that we can even get such a $\left(\mathcal{G}^{\prime}, k^{\prime}\right)$ with $k^{\prime}=k$ in time polynomial in $|V|^{r / k}$, and hence by (4) and (5), in time polynomial in $m$. Note that by (4) and (2) the assumptions of the lemma are satisfied.

Lemma 3. There is an algorithm that assigns to every graph $\mathcal{G}=(V, E)$ and $r, k \in \mathbb{N}$ with

$$
k \leq r \leq|V| \text { and } 2 \leq|V|
$$

a graph $\mathcal{G}^{\prime}$ in time polynomial in $|V|^{r / k}$ such that

$$
\mathcal{G} \text { has a dominating set of size } r \quad \Longleftrightarrow \quad \mathcal{G}^{\prime} \text { has a dominating set of size } k \text {. }
$$

Proof: Assume that $\mathcal{G}=(V, E)$ is a graph, $k \leq r \leq|V|$, and $2 \leq|V|$. We choose the uniquely determined $q$ and $s$ in $\mathbb{N}$ such that

$$
k \cdot q=r+s \text { with } 0 \leq s<k .
$$

We first add $s$ new isolated vertices to $\mathcal{G}$ thus obtaining a graph $\mathcal{G}_{0}=\left(V_{0}, E_{0}\right)$ such that

$$
\mathcal{G} \text { has a dominating set of size } r \quad \Longleftrightarrow \quad \mathcal{G}_{0} \text { has a dominating set of size } k \cdot q \text {. }
$$


Then $\left|V_{0}\right|=|V|+s \leq 2|V|$. Let $\left[V_{0}\right]^{q}$ be the set of subsets of $V_{0}$ of $q$ elements. Let the graph $\mathcal{G}^{\prime}$ have the set of vertices

$$
V^{\prime}:=V_{0} \cup\left[V_{0}\right]^{q}
$$

and as edges the edges in $E_{0}$, the edge $\{X, Y\}$ for every distinct $X, Y \in\left[V_{0}\right]^{q}$, and edges $\{u, X\}$ for $u \in V_{0}$ and $X \in\left[V_{0}\right]^{q}$ such that

$$
u \in X \text { or there is } v \in X \text { with }\{u, v\} \in E_{0} .
$$

Note that $\mathcal{G}^{\prime}$ can be constructed from $\mathcal{G}$ in time polynomial in $|V|^{q}$ and hence in time polynomial in $|V|^{r / k}$.

Furthermore (in this yields the desired equivalence in view of (6))

$$
\mathcal{G}_{0} \text { has a dominating set of size } k \cdot q \quad \Longleftrightarrow \quad \mathcal{G}^{\prime} \text { has a dominating set of size } k \text {. }
$$

In fact, if $\mathcal{G}_{0}$ has a dominating $D$ set of size $k \cdot q$, then we partition $D$ into $k$ sets $X_{1}, \ldots, X_{k}$ all of size $q$. One easily verifies that $\left\{X_{1}, \ldots, X_{k}\right\}$ is a dominating set of $\mathcal{G}^{\prime}$. Conversely, assume that $\mathcal{G}^{\prime}$ has a dominating set of size $k$ consisting of $X_{1}, \ldots, X_{\ell} \in\left[V_{0}\right]^{q}$ and $u_{1}, \ldots, u_{m} \in V_{0}$. Then

$$
D:=X_{1} \cup \ldots \cup X_{\ell} \cup\left\{u_{1}, \ldots, u_{m}\right\}
$$

is a dominating set of $\mathcal{G}_{0}$ of size $\leq k \cdot q$; hence there is a dominating set of size $k \cdot q$.

As $p$-Dominating-SeT is $\mathrm{W}[2]$-complete under fpt-reductions, we get from Theorem 2 and the Miniaturization Theorem:

Corollary 4. W[2] = FPT if and only if there is an algorithm deciding whether a graph $\mathcal{G}=(V, E)$ has a dominating set of size $k$ in time $2^{{ }^{\text {eff }}(k \cdot \log |V|)} \cdot|V|^{O(1)}$.

\section{The general framework}

We show that the result obtained in the previous section for the dominating set problem can be generalized to any parameterized problem satisfying an analogue of Lemma 3.

We start with a simple observation that often will be useful.

Proposition 5. Let $(Q, \kappa)$ be a parameterized problem over the alphabet $\Sigma$ and $\kappa^{\prime}: \Sigma^{*} \rightarrow \mathbb{N}$ a further parameterization of $Q$ such that $\kappa^{\prime}(x) \leq O(\kappa(x) \cdot \log |x|)$. Then:

$$
(Q, \kappa) \leq{ }^{\mathrm{fpt}} \mathscr{M}\left(Q, \kappa^{\prime}\right) .
$$

In particular,

$$
(Q, \kappa) \leq{ }^{\mathrm{fpt}} \mathscr{M}(Q, \kappa) .
$$

Proof: Immediate as the function $x \mapsto(x,|x|)$ is an fpt-reduction from $(Q, \kappa)$ to $\mathscr{M}\left(Q, \kappa^{\prime}\right)$.

Corollary 6. Let $(Q, \kappa)$ be a parameterized problem over the alphabet $\Sigma$ and $\kappa^{\prime}$ a further parameterization such that $\kappa^{\prime}(x) \leq O(\kappa(x) \cdot \log |x|)$. Furthermore let $\mathrm{C}$ be a class of parameterized problems.

If $(Q, \kappa)$ is $\mathrm{C}$-complete under fpt-reductions and $\mathscr{M}\left(Q, \kappa^{\prime}\right) \in \mathrm{C}$, then $\mathscr{M}\left(Q, \kappa^{\prime}\right)$ is $\mathrm{C}$ complete under fpt-reductions.

Now we turn to a generalization of the results of the previous section.

Definition 7. Let $(Q, \kappa)$ be a parameterized problem over the alphabet $\Sigma$. Let $h: \Sigma^{*} \rightarrow \mathbb{N}$ be a function. We say that $(Q, \kappa)$ has the $h$-condensation property if there are a computable function $f: \mathbb{N} \rightarrow \mathbb{N}$ and an algorithm that for every $x \in \Sigma^{*}$ and $k \in \mathbb{N}$ with $1 \leq k \leq \kappa(x)$ computes an $x^{\prime} \in \Sigma^{*}$ in time polynomial in

$$
h(x)^{\kappa(x) / k}+|x|
$$

such that 


$$
\begin{aligned}
& -x \in Q \Longleftrightarrow x^{\prime} \in Q ; \\
& -\kappa\left(x^{\prime}\right) \leq f(k) .
\end{aligned}
$$

In most examples we can choose as $f$ the identity function (and then we do not mention $f$ explicitly). In these cases the last condition turns into

$$
-\kappa\left(x^{\prime}\right) \leq k .
$$

In [3] the authors introduce the notion of scalable parameterized problem and prove Theorem 10 below for scalable problems. The reader familiar with that paper will easily show that a parameterized problem is scalable if and only if it has the $h$-condensation property for $h(x):=|x|$ and we can choose as $f$ the identity function.

Examples 8. (a) Lemma 3 shows that $p$-DOMINATING-SET has the $h$-condensation property for the function $h$ given by $h(\mathcal{G}, r):=|V| .^{3}$

(b) $p$-INDEPENDENT-SET has the $h$-condensation property for $h$ with $h(\mathcal{G}, r):=|V|$. The verification is similar to the case of the dominating set problem and is implicit in [3].

(c) The parameterized problem:

$\begin{aligned} & p \text {-RED/BLUE-NONBLOCKER } \\ & \text { Instance: } \quad \text { A graph } \mathcal{G}=(V, E), \text { a partition of } V \text { into two sets } R \text { and } \\ & B \text { (the red and the blue vertices, respectively), and } r \in \mathbb{N} . \\ & \text { Parameter: } r . \\ & \text { Question: } \begin{array}{l}\text { Does there exist a set } S \text { of size } r \text { of red vertices such that } \\ \text { every blue vertex has at least one red neighbor that does } \\ \text { not belong to } S ?\end{array}\end{aligned}$

has the $h$-condensation property for the function $h$ given by $h(\mathcal{G}, R, B, r):=|R|$.

In fact, let $(\mathcal{G}, R, B, r)$ be an instance of the problem and let $k \in \mathbb{N}$ with $1 \leq k \leq r$. By adding less than $k$ isolated vertices to the red part we can assume that $k$ divides $r$, say, $q \cdot k=r$. Now we pass to the graph $\mathcal{G}^{\prime}=\left(V^{\prime}, E^{\prime}\right)$ with $V^{\prime}=R^{\prime} \cup B^{\prime}$. Here, $R^{\prime}$, the set of its red vertices, is $[R]^{q}$, the set of subsets of $R$ of size $q$, and $B^{\prime}$ contains the old blue vertices and for every pair $\{X, Y\}$ of distinct elements of $[R]^{q}$ with nonempty intersection a vertex $b_{\{X, Y\}}$. That is,

$$
B^{\prime}:=B \cup\left\{b_{\{X, Y\}} \mid X, Y \in[R]^{q} \text { and } X \cap Y \neq \emptyset\right\} .
$$

Furthermore, let

$$
\begin{aligned}
E^{\prime}:= & \left\{\{b, X\} \mid b \in B, X \in[R]^{q}, \text { and }\{b, a\} \in E \text { for some } a \in X\right\} \\
& \cup\left\{\left\{b_{\{X, Y\}}, X\right\} \mid X, Y \in[R]^{q} \text { and } X \cap Y \neq \emptyset\right\} .
\end{aligned}
$$

One easily verifies that $(\mathcal{G}, R, B, r)$ is a positive instance of $p$-RED/BLUE-NONBLOCKER if and only if $\left(\mathcal{G}^{\prime}, R^{\prime}, B^{\prime}, k\right)$ is. Moreover, $\left(\mathcal{G}^{\prime}, R^{\prime}, B^{\prime}, k\right)$ can be obtained in time polynomial in $|R|^{r / k}+|V|$.

Further examples will be given in the next sections.

Definition 9. Let $(Q, \kappa)$ be a parameterized problem over the alphabet $\Sigma$. Let $h: \Sigma^{*} \rightarrow \mathbb{N}$ be a function computable in polynomial time. The h-reparameterization $\left(Q, \kappa_{h}\right)$ of $(Q, \kappa)$ is then given by

$$
\kappa_{h}(x):=\kappa(x) \cdot \log h(x) .
$$

The next result shows that $h$-reparameterizations of problems $(Q, \kappa)$ with the $h$-condensation property are preimages of $(Q, \kappa)$.

\footnotetext{
${ }^{3}$ Lemma 3 only shows that the relevant inequality holds for $k \neq 1$. As usual, the failure for finitely many values is not relevant here and thus we do not mention it in the following.
} 
Theorem 10. Let $(Q, \kappa)$ be a parameterized problem over $\Sigma$. Let $h: \Sigma^{*} \rightarrow \mathbb{N}$ be a function computable in polynomial time such that $h(x) \leq|x|$ for all $x \in \Sigma^{*}$. Furthermore assume that $(Q, \kappa)$ has the $h$-condensation property. Then

$$
\mathscr{M}\left(Q, \kappa_{h}\right) \equiv^{\mathrm{fpt}}(Q, \kappa) .
$$

Proof: $(Q, \kappa) \leq^{\mathrm{fpt}} \mathscr{M}\left(Q, \kappa_{h}\right):$ As $h(x) \leq|x|$, we have

$$
\kappa_{h}(x)=\kappa(x) \cdot \log h(x) \leq \kappa(x) \cdot \log |x| ;
$$

thus the result follows from Proposition 5.

$\mathscr{M}\left(Q, \kappa_{h}\right) \leq^{\mathrm{fpt}}(Q, \kappa):$ Let $(x, m)$ be an instance of $\mathscr{M}\left(Q, \kappa_{h}\right)$. The parameter of $(x, m)$ is

$$
k:=\left\lceil\frac{\kappa_{h}(x)}{\log m}\right\rceil=\left\lceil\frac{\kappa(x) \cdot \log h(x)}{\log m}\right\rceil .
$$

- If $h(x)>m$, then $k>\kappa(x)$. Hence, $x$ is an instance of $Q$ equivalent to $(x, m)$; its parameter $\kappa(x)$ is bounded by $k$, the parameter of $(x, m)$.

- If $h(x) \leq m$, then $k \leq \kappa(x)$. Furthermore, $\kappa(x) \cdot \log h(x) \leq k \cdot \log m$ and thus,

$$
h(x)^{\kappa(x) / k} \leq m .
$$

As $(Q, \kappa)$ has the $h$-condensation property, we find in time polynomial in $h(x)^{\kappa(x) / k}+$ $|x|$ an instance $x^{\prime}$ such that $\left(x \in Q \Longleftrightarrow x^{\prime} \in Q\right.$ ) and $\kappa\left(x^{\prime}\right) \leq f(k)$ (where $f$ is the computable function according to Definition 7). Since $h(x)^{\kappa(x) / k}+|x| \leq m+|x|$, the time required is actually polynomial in $m+|x|$.

Altogether, the function

$$
R(x, m):= \begin{cases}x & h(x)>m, \\ x^{\prime} & h(x) \leq m,\end{cases}
$$

is an fpt-reduction from $\mathscr{M}\left(Q, \kappa_{h}\right)$ to $(Q, \kappa)$.

Corollary 11. Let $(Q, \kappa)$ be a parameterized problem over $\Sigma$. Let $h: \Sigma^{*} \rightarrow \mathbb{N}$ be a function computable in polynomial time such that $h(x) \leq|x|$ for all $x \in \Sigma^{*}$. Furthermore assume that $(Q, \kappa)$ has the $h$-condensation property. Then

$$
\begin{aligned}
& (Q, \kappa) \text { is fixed-parameter tractable } \\
& \qquad \Longleftrightarrow x \in Q \text { is solvable in time } 2^{o^{\text {eff }}(\kappa(x) \cdot \log h(x))} \cdot|x|^{O(1)} .
\end{aligned}
$$

Example 8(c) together with Theorem 10 yield:

Proposition 12. $\quad \mathscr{M}($ red-Red/BLue-NonblockeR $) \equiv^{\mathrm{fpt}} p$-ReD/Blue-Nonblocker, where the problem red-RED/BLUE-NONBLOCKER is the reparameterization of $p$-RED/BLUENONBLOCKER obtained by changing the parameter to $r \cdot \log |R|$.

\section{Further applications}

In this section we give applications to weighted satisfiability problems for formulas of propositional logic and Boolean circuits. First we recall some notions thereby using the notations from [8].

We denote the class of Boolean circuits by CIRC and the class of propositional formulas by PROP. For $t \geq 0$ and $d \geq 1$ we inductively define the following classes $\Gamma_{t, d}$ and $\Delta_{t, d}$ of 
formulas:

$$
\begin{aligned}
\Gamma_{0, d} & :=\left\{\lambda_{1} \wedge \ldots \wedge \lambda_{s} \mid s \in[d], \lambda_{1}, \ldots, \lambda_{s} \text { literals }\right\}, \\
\Delta_{0, d} & :=\left\{\lambda_{1} \vee \ldots \vee \lambda_{s} \mid s \in[d], \lambda_{1}, \ldots, \lambda_{s} \text { literals }\right\}, \\
\Gamma_{t+1, d} & :=\left\{\bigwedge_{i \in I} \delta_{i} \mid I \text { a finite nonempty index set and } \delta_{i} \in \Delta_{t, d} \text { for all } i \in I\right\}, \\
\Delta_{t+1, d} & :=\left\{\bigvee_{i \in I} \gamma_{i} \mid I \text { a finite nonempty index set and } \gamma_{i} \in \Gamma_{t, d} \text { for all } i \in I\right\} .
\end{aligned}
$$

That is, in propositional formulas we distinguish between small conjunctions, denoted by $\wedge$, which are just conjunctions of two formulas, and big conjunctions, denoted by $\Lambda$, which are conjunctions of finite sets of formulas. Analogously, we distinguish between small disjunctions, $\vee$, and big disjunctions, $\bigvee$.

If in the definition of $\Gamma_{0, d}$ and $\Delta_{0, d}$ we require that all literals are positive (negative), then we obtain the sets denoted by $\Gamma_{t, d}^{+}$and $\Delta_{t, d}^{+}\left(\Gamma_{t, d}^{-}\right.$and $\left.\Delta_{t, d}^{-}\right)$, respectively.

Let $V$ be a set of propositional variables. Often we tacitly identify an assignment $S: V \rightarrow$ \{TRUe, FALSE $\}$ with the set $\{X \in V \mid S(X)=$ TRUE $\}$. The weight of an assignment $S$ is $|S|$, the number of variables set to TRUE. A propositional formula $\alpha$ is $k$-satisfiable (where $k \in \mathbb{N}$ ), if there is an assignment for the set $\operatorname{var}(\alpha)$ of variables of $\alpha$ of weight $k$ satisfying $\alpha$. Similarly we define the $k$-satisfiability of a circuit. For a set $\Gamma$ of propositional formulas or circuits the parameterized weighted satisfiability problem for $\Gamma$ is defined by

$$
\begin{aligned}
p \text {-WSAT }(\Gamma) & \\
\text { Instance: } & \gamma \in \Gamma \text { and } k \in \mathbb{N} . \\
\text { Parameter: } & k . \\
\text { Question: } & \text { Does } \gamma \text { have a satisfying assignment of weight } k ?
\end{aligned}
$$

We shall use the following result (which we also can read as definition of the classes $\mathrm{W}[1]$, $\mathrm{W}[2], \ldots$ of the W-hierarchy and of the classes $\mathrm{W}[\mathrm{SAT}]$ and $\mathrm{W}[\mathrm{P}])$.

Theorem 13. Let $t, d \in \mathbb{N}$ with $t+d \geq 3$.

- If $t$ is even, then $p$ - $\mathrm{WSAT}\left(\Gamma_{t, d}^{+}\right)$is $\mathrm{W}[t]$-complete under fpt-reductions.

- If $t$ is odd, then $p$ - $\mathrm{WSAT}\left(\Gamma_{t, d}^{-}\right)$is $\mathrm{W}[t]$-complete under fpt-reductions.

- p-WSAT(PROP) is W[SAT]-complete under fpt-reductions.

- $p$-WSAT(CIRC) is W[P]-complete under fpt-reductions.

Again let $\Gamma$ be a set of propositional formulas or circuits. We shall consider the $h$-reparameterizations var-WSAT $(\Gamma)$ and form-WSAT $(\Gamma)$ of $p-\operatorname{WSAT}(\Gamma)$ obtained for $h(\gamma, r):=$ $|\operatorname{var}(\gamma)|(=$ number of variables of $\gamma)$ and $h(\gamma, r):=|\gamma|(=$ size of $\gamma)$, respectively; that is,

$$
\begin{aligned}
\text { var-WSAT }(\Gamma) & \\
\text { Instance: } & \gamma \in \Gamma \text { and } r \in \mathbb{N} . \\
\text { Parameter: } & r \cdot \log |\operatorname{var}(\gamma)| . \\
\text { Question: } & \text { Does } \gamma \text { have a satisfying assignment of weight } r ?
\end{aligned}
$$

and

$$
\begin{aligned}
\text { form-WSAT }(\Gamma) & \\
\text { Instance: } & \gamma \in \Gamma \text { and } r \in \mathbb{N} . \\
\text { Parameter: } & r \cdot \log |\gamma| . \\
\text { Question: } & \text { Does } \gamma \text { have a satisfying assignment of weight } r \text { ? }
\end{aligned}
$$

For relevant classes the first function $h$ has the condensation property: 
Example 14. For the function $h$ given by $h(\gamma, r):=|\operatorname{var}(\gamma)|$ the problem $p-\mathrm{WSAT}(\Gamma)$ has the $h$-condensation property for

- for $\Gamma:=\Gamma_{t, d}^{+}$with even $t \geq 1$ and $d \geq 1$

- for $\Gamma:=\Gamma_{t, d}^{-}$with odd $t \geq 1, d \geq 1$ and $t+d \geq 3$

- for $\Gamma:=$ PROP and for $\Gamma:=$ CIRC.

We show this for $\Gamma:=\Gamma_{t, d}^{+}$with even $t$. Consider an instance $(\alpha, r)$ of $p$-WSAT $\left(\Gamma_{t, d}^{+}\right)$and let $1 \leq k \leq r \leq|\operatorname{var}(\alpha)|$. We choose the uniquely determined $q, s \in \mathbb{N}$ such that

$$
k \cdot q=r+s \text { with } 0 \leq s<k .
$$

We take $s$ new variables $Y_{1}, \ldots, Y_{s}$ and let

$$
\alpha_{0}:=\alpha \wedge \bigwedge_{i \in[s]} Y_{i}
$$

It is easy to see that $\alpha_{0}$ is in $\Gamma_{t, d}^{+}$, up to logical equivalence, and that

$$
(\alpha, r) \in p-\mathrm{WSAT}\left(\Gamma_{t, d}^{+}\right) \quad \Longleftrightarrow \quad\left(\alpha_{0}, r+s\right) \in p \text {-WSAT }\left(\Gamma_{t, d}^{+}\right) .
$$

Let $\left[\operatorname{var}\left(\alpha_{0}\right)\right]^{q}$ be the set of subsets of $\operatorname{var}\left(\alpha_{0}\right)$ of size $q$. For each $\mathcal{Y} \in\left[\operatorname{var}\left(\alpha_{0}\right)\right]^{q}$ let $X(\mathcal{Y})$ be a new variable. Now we replace each variable $X$ in $\alpha_{0}$ by

$$
\bigvee_{\substack{\mathcal{Y} \in\left[\operatorname{var}\left(\alpha_{0}\right)\right]^{q} \\ \text { with } X \in \mathcal{Y}}} X(\mathcal{Y}) .
$$

As $t$ is even, the formula thus obtained is equivalent to a formula $\alpha^{\prime}$ in $\Gamma_{t, d}^{+}$. Altogether, we obtain $\alpha^{\prime}$ in time polynomial in $h(\alpha, r)^{r / k}+|\alpha|$ (recall that $h(\alpha, r)=|\operatorname{var}(\alpha)|$ and note that $\alpha_{0}$ has at most $2 h(\alpha, r)$ variables and that $\left.q=(r+s) / k \leq r / k+1\right)$. Using the fact that $\alpha_{0}$ is monotone, it is routine to verify

$$
\left(\alpha_{0}, r+s\right) \in p \text { - } \mathrm{WSAT}\left(\Gamma_{t, d}^{+}\right) \Longleftrightarrow\left(\alpha^{\prime}, k\right) \in p \text {-WSAT }\left(\Gamma_{t, d}^{+}\right) .
$$

Now (7) and (8) yield the desired equivalence.

As already remarked in [3]:

Lemma 15. Let $t, d \in \mathbb{N}$ with $t+d \geq 3$. Then for even $t$

$$
\operatorname{var}-\mathrm{WSAT}\left(\Gamma_{t, d}\right) \equiv^{\mathrm{serf}} \operatorname{var}-\mathrm{WSAT}\left(\Gamma_{t, d}^{+}\right)
$$

and for odd $t$

$$
\operatorname{var}-\mathrm{WSAT}\left(\Gamma_{t, d}\right) \equiv{ }^{\text {serf }} \operatorname{var}-\mathrm{WSAT}\left(\Gamma_{t, d}^{-}\right) .
$$

Proof: Let $t$ be even. In [9], the authors exhibit a polynomial time reduction $R$ from the problem $p$-WSAT $\left(\Gamma_{t, d}\right)$ to $p$ - $\mathrm{WSAT}\left(\Gamma_{t, d}^{+}\right)$, which has the following property: Let $\alpha \in \Gamma_{t, d}$ and $1 \leq k \leq n$, where $n$ is the number of variables of $\alpha$. If $R(\alpha, k)=\left(\alpha^{\prime}, k^{\prime}\right)$ and $\alpha^{\prime}$ has $n^{\prime}$ variables, then

$$
k^{\prime}=O(k) \quad \text { and } \quad n^{\prime}=n^{O(1)}
$$

Hence

$$
k^{\prime} \cdot \log n^{\prime}=O(k \cdot \log n) .
$$

Thus $R$ is a serf-reduction from $\operatorname{var}-\operatorname{WSAT}\left(\Gamma_{t, d}\right)$ to $\operatorname{var}-\operatorname{WSAT}\left(\Gamma_{t, d}^{+}\right)$. Obviously we have $\operatorname{var}-\mathrm{WSAT}\left(\Gamma_{t, d}^{+}\right) \leq^{\text {serf }} \operatorname{var}-\mathrm{WSAT}\left(\Gamma_{t, d}\right)$, so we get the claimed equivalence. [9].

For odd $t$, we use the corresponding reduction from $p$ - $\mathrm{WSAT}\left(\Gamma_{t, d}\right)$ to $p-\mathrm{WSAT}\left(\Gamma_{t, d}^{-}\right)$of

The following theorem yields problems complete under serf-reductions in the preimage of
$\mathrm{W}[t]$. 
Theorem 16. Let $t, d \in \mathbb{N}$ with $t+d \geq 3$. If $t$ is even, then the following problems are $\mathrm{W}[t]$-complete under fpt-reductions:

(1) $\mathscr{M}\left(\operatorname{var}-\mathrm{WSAT}\left(\Gamma_{t, d}\right)\right)$;

(2) $\mathscr{M}\left(\operatorname{var}-\mathrm{WSAT}\left(\Gamma_{t, d}^{+}\right)\right)$;

(3) $\mathscr{M}\left(\right.$ form-WSAT $\left.\left(\Gamma_{t, d}\right)\right)$;

(4) $\mathscr{M}\left(\right.$ form-WSAT $\left.\left(\Gamma_{t, d}^{+}\right)\right)$.

For odd t one has to replace $\Gamma_{t, d}^{+}$by $\Gamma_{t, d}^{-}$.

Proof: Let $t$ be even. By Example 14 and Theorem 10 we know that

$$
\mathscr{M}\left(\operatorname{var}-\mathrm{WSAT}\left(\Gamma_{t, d}^{+}\right)\right) \equiv{ }^{\mathrm{fpt}} p-\mathrm{WSAT}\left(\Gamma_{t, d}^{+}\right) .
$$

As $p$ - $\mathrm{WSAT}\left(\Gamma_{t, d}^{+}\right)$is $\mathrm{W}[t]$-complete under fpt-reductions, this yields part (2). Part (1) follows from part (2) by the preceding lemma and the Miniaturization Theorem.

Clearly, form-WSAT $(\Gamma) \leq$ serf $v a r$-WSAT $(\Gamma)$ for every $\Gamma$. Hence

$$
p \text {-WSAT }(\Gamma) \leq^{\mathrm{fpt}} \mathscr{M}(\text { form-WSAT }(\Gamma)) \leq^{\mathrm{fpt}} \mathscr{M}(\operatorname{var}-\mathrm{WSAT}(\Gamma)),
$$

where the first $\leq{ }^{\mathrm{fpt}}$ holds by Proposition 5. Now parts (3) and (4) follow from parts (1) and (2).

Similarly one gets:

Corollary 17. (1) The problems $\mathscr{M}($ var-WSAT(PROP)) and $\mathscr{M}($ form-WSAT(PROP)) are $\mathrm{W}[\mathrm{SAT}]$-complete under fpt-reductions.

(2) The problems $\mathscr{M}($ var-WS AT(CIRC)) and $\mathscr{M}($ form-WSAT(CIRC)) are $\mathrm{W}[\mathrm{P}]$-complete under fpt-reductions.

The previous results have the following surprising consequence.

Corollary 18. Let $t, d \in \mathbb{N}$ with $t+d \geq 3$. Let $\Gamma:=\Gamma_{t, d}$, or $\Gamma:=\Gamma_{t, d}^{+}$for even $t$, or $\Gamma:=\Gamma_{t, d}^{-}$for odd $t$, or $\Gamma:=$ PROP, or $\Gamma:=$ CIRC. Then

$$
\operatorname{var}-\mathrm{WSAT}(\Gamma) \equiv^{\mathrm{serf}} \text { form-WSAT }(\Gamma) .
$$

We do not know for what other sets $\Gamma$ this equivalence holds.

Note that the well-known [5] $(k \cdot \log n)$-trick and its "reverse" can be reformulated (or at least imply)

$$
v a r-\mathrm{WSAT}(\mathrm{PROP}) \equiv{ }^{\mathrm{serf}} p-\mathrm{SAT}(\mathrm{PROP}) \quad \text { and } \quad v a r-\mathrm{WSAT}(\mathrm{CIRC}) \equiv{ }^{\text {serf }} p-\mathrm{SAT}(\mathrm{CIRC}),
$$

where $p-\operatorname{SAT}(\Gamma)$ denotes the satisfiability problem for $\Gamma$ parameterized by the number of variables. From Corollary 17 we obtain the following result first derived in [1] (also see [5]):

Corollary 19. (1) $\mathscr{M}(p-\mathrm{SAT}(\mathrm{PROP}))$ is $\mathrm{W}[\mathrm{SAT}]$-complete under fpt-reductions.

(2) $\mathscr{M}(p-\mathrm{SAT}(\mathrm{CIRC}))$ is $\mathrm{W}[\mathrm{P}]$-complete under fpt-reductions.

Recall that the classes M[1], M[2],... of the M-hierarchy M-hierarchy and the classes $\mathrm{M}[\mathrm{SAT}]$ and $\mathrm{M}[\mathrm{P}]$ are defined by

- M $[t]$ is the class of parameterized problems fpt-reducible to $\mathscr{M}\left(p-\operatorname{SAT}\left(\Gamma_{t, d}\right)\right)$ for some $d \geq 1$ :

- M[SAT $]$ is the class of parameterized problems fpt-reducible to $\mathscr{M}(p$-SAT(PROP));

- M[P] is the class of parameterized problems fpt-reducible to $\mathscr{M}(p-\mathrm{SAT}(\mathrm{CIRC}))$. 
Furthermore, the following relationship between the classes of the M-hierarchy and the classes of the W-hierarchy holds: for every $t \geq 1, \mathrm{M}[t] \subseteq \mathrm{W}[t] \subseteq \mathrm{M}[t+1]$, moreover, $\mathrm{M}[\mathrm{SAT}]=$ $\mathrm{W}[\mathrm{SAT}]$ and $\mathrm{M}[\mathrm{P}]=\mathrm{W}[\mathrm{P}]$ (see $[6,8]$ ).

Part (2) of the following theorem is due to Chen et al. [2].

Theorem 20. Let $t, d \in \mathbb{N}$ with $t+d \geq 3$.

(1) If $\mathrm{W}[t]=\mathrm{FPT}$, then one can decide $p$ - $\mathrm{WSAT}\left(\Gamma_{t, d}\right)$ in time $|\operatorname{var}(\alpha)|^{\left.\right|^{\text {eff }}(k)} \cdot|\alpha|^{O(1)}$ for every instance $(\alpha, k)$.

(2) If $\mathrm{M}[t] \neq \mathrm{FPT}$, then one cannot decide $p$-WSAT $\left(\Gamma_{t, d}\right)$ in time $|\operatorname{var}(\alpha)|^{o^{\text {eff }}(k)} \cdot|\alpha|^{O(1)}$ for every instance $(\alpha, k)$.

Proof: To prove (1) we assume that $\mathrm{W}[t]=$ FPT. Then, by Theorem 16 (1), we have $\mathscr{M}\left(\operatorname{var}-\mathrm{WSAT}\left(\Gamma_{t, d}\right)\right) \in \mathrm{FPT}$ and hence, by the Miniaturization Theorem, var-WSAT$\left(\Gamma_{t, d}\right) \in$ SUBEPT. Therefore for some computable, unbounded and nondecreasing function $\iota$ there is an algorithm that, for any $\Gamma_{t, d}$-formula $\alpha$ and $k \in \mathbb{N}$, decides whether $\alpha$ has a satisfying assignment of weight $k$ in time

$$
2^{(k \cdot \log |\operatorname{var}(\alpha)|) / \iota(k \cdot \log |\operatorname{var}(\alpha)|)} \cdot|\alpha|^{O(1)} .
$$

We get our claim as

$$
\begin{aligned}
& 2^{(k \cdot \log |\operatorname{var}(\alpha)|) / \iota(k \cdot \log |\operatorname{var}(\alpha)|)} \cdot|\alpha|^{O(1)} \\
& \quad=|\operatorname{var}(\alpha)|^{k / \iota(k \cdot \log |\operatorname{var}(\alpha)|)} \cdot|\alpha|^{O(1)}=|\operatorname{var}(\alpha)|^{\text {off }^{\text {eff }}(k)} \cdot|\alpha|^{O(1)} .
\end{aligned}
$$

As already remarked the reader can find a proof of (2) in [2].

By the same method we get:

Theorem 21. $\quad-\mathrm{W}[\mathrm{SAT}]=\mathrm{FPT}$ if and only if one can decide $p$ - $\mathrm{WSAT}(\mathrm{PROP})$ in time $|\operatorname{var}(\alpha)|^{o^{\text {eff }}(k)} \cdot|\alpha|^{O(1)}$ for every instance $(\alpha, k)$.

$-\mathrm{W}[\mathrm{P}]=\mathrm{FPT}$ if and only if one can decide $p$-WS AT $(\mathrm{CIRC})$ in time $|\operatorname{var}(\alpha)|^{\left.\right|^{\text {eff }}(k)} \cdot|\alpha|^{O(1)}$ for every instance $(\alpha, k)$.

\section{Fagin-definable problems}

In this section we consider Fagin-definable problems. We first recall their definition and fix our notation for first-order logic.

A vocabulary $\tau$ is a finite set of relation symbols. Each relation symbol has an arity. The arity of a vocabulary is the maximum of the arities of its symbols. As all vocabularies contain the binary equality symbol $=$, we do not mention it explicitly. A $\tau$-structure $\mathcal{A}$ consists of a set $A$ called the universe, which we assume to be finite, and an interpretation $R^{\mathcal{A}} \subseteq A^{r}$ of each $r$-ary relation symbol $R \in \tau$. For example, we view a graph as a structure $\mathcal{G}=\left(G, E^{\mathcal{G}}\right)$, where $E$ is a binary relation symbol and $E^{\mathcal{G}}$ is an irreflexive and symmetric binary relation on the set $G$ of vertices. Nevertheless, as in the previous sections, we often denote the vertex set of a graph $\mathcal{G}$ by $V$ and the edge set by $E$ (instead of $G$ and $E^{\mathcal{G}}$ ) and use the set notation $\{v, w\}$ for edges.

For a $\tau$-structure $\mathcal{A}$ we denote by $\|\mathcal{A}\|$ its size, that is, the length of a string encoding $\mathcal{A}$ in a natural way. The number $\|\mathcal{A}\|$ will be within a polynomial factor of the term

$$
|\tau|+|A|+\sum_{R \in \tau}\left|R^{\mathcal{A}}\right| \cdot \operatorname{arity}(R) .
$$

Formulas of first-order logic of vocabulary $\tau$ are built up from atomic formulas $x=y$ and $R x_{1} \ldots x_{r}$ where $x, y, x_{1}, \ldots, x_{r}$ are variables and $R \in \tau$ is of arity $r$, using the boolean connectives and existential and universal quantification. For $t \geq 0$ let $\Pi_{t}$ denote the class of all first-order formulas of the form

$$
\forall x_{11} \ldots \forall x_{1 k_{1}} \exists x_{21} \ldots \exists x_{2 k_{2}} \ldots Q x_{t 1} \ldots Q x_{t k_{t}} \psi
$$


where $Q=\exists$ if $t$ is even and $Q=\forall$ otherwise, and where $\psi$ is quantifier-free. $\Sigma_{t}$-formulas are defined analogously starting with a block of existential quantifiers.

Let $\varphi(X)$ be any first-order formula with a (second-order) set variable $X$ (and with no other free variables). It Fagin-defines the parameterized problem (cf. [8] to see where the notation $p-\mathrm{WD}_{\varphi}$ comes from and what is the relationship to Fagin's descrpitive characterization of NP).

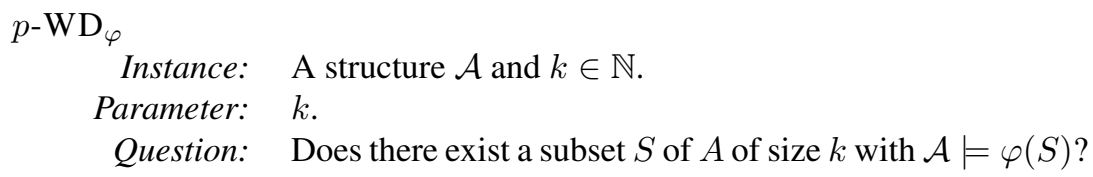

Here $\mathcal{A}=\varphi(S)$ means that $\varphi(X)$ holds in $\mathcal{A}$ if $X$ is interpreted by $S$. Its canonical reparameterization is the problem

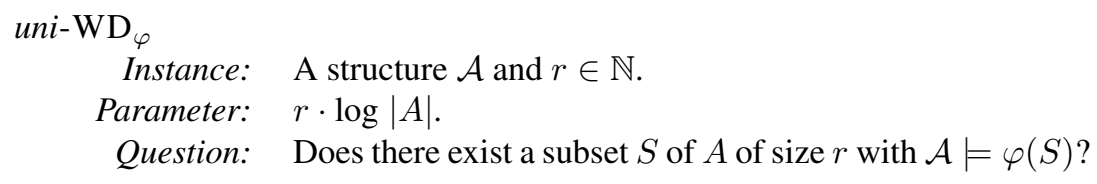

Our main result reads as follows:

Theorem 22. Let $\varphi(X)$ be a $\Pi_{t}$-formula and assume that $p-\mathrm{WD}_{\varphi}$ is $\mathrm{W}[t]$-complete under fpt-reductions. Then $\mathscr{M}\left(\right.$ uni- $\left.\mathrm{WD}_{\varphi}\right)$ is $\mathrm{W}[t]$-complete under fpt-reductions.

This result has many applications. For example, the problems $p$-CLIQUE and $p$-SET-PACKING are $\mathrm{W}[1]$-complete problems Fagin-definable by $\Pi_{1}$-formulas and the problems $p$-TOURNAMENT-DOMINATING-SET, $p$-KERNEL are W[2]-complete problems Fagin-definable by $\Pi_{2}$ formulas. Thus the miniaturizations of their canonical reparameterizations are $\mathrm{W}[1]$-complete and $\mathrm{W}[2]$-complete, respectively (cf. [8] for the definitions of the problems).

Before we turn to a proof of Theorem 22, we consider an example which suggests to prove a more general result. HitTING-SET is the problem:

HitTING-SET

Instance: A hypergraph $\mathcal{H}=(V, E)$ and $r \in \mathbb{N}$.

Question: Does there exist a set $S \subseteq V$ of size $r$ such that $S \cap e \neq \emptyset$

for all $e \in E$ ?

Its canonical parameterization is:

vert-HitTING-SET

Instance: A hypergraph $\mathcal{H}=(V, E)$ and $r \in \mathbb{N}$.

Parameter: $\quad r \cdot \log |V|$.

Question: Does there exist a set $S \subseteq V$ of size $r$ such that $S \cap e \neq \emptyset$ for all $e \in E$ ?

In order to rewrite the problem as a Fagin-definable one, we represent a hypergraph $\mathcal{H}$ as a

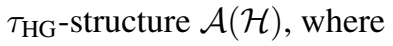

$$
\tau_{\mathrm{HG}}=\{V E R T, E D G E, I\}
$$

with unary relation symbols $V E R T$ and $E D G E$ and binary relation symbol $I$ : We let

$$
A(\mathcal{H}):=V \cup E, \quad V E R T^{\mathcal{A}(\mathcal{H})}:=V, \quad \operatorname{EDGE}^{\mathcal{A}(\mathcal{H})}:=E,
$$

and

$$
I^{\mathcal{A}(\mathcal{H})}:=\{(v, e) \mid v \in V, e \in E \text {, and } v \in e\} .
$$


The following formula expresses that $X$ is a hitting set:

$$
\operatorname{hit}_{0}(X):=\forall x \exists y((X x \rightarrow \operatorname{VERT} x) \wedge(E D G E x \rightarrow(X y \wedge \text { Iyx }))) .
$$

Furthermore, it is not hard to see that there is a $\Pi_{2}$-sentence hyp of vocabulary $\tau_{\mathrm{HG}}$, which is satisfied exactly by those $\tau_{\mathrm{HG}}$-structures that, up to isomorphism, have the form $\mathcal{A}(\mathcal{H})$ for some hypergraph $\mathcal{H}$. We set

$$
\operatorname{hit}(X):=\operatorname{hyp} \wedge \operatorname{hit}_{0}(X) .
$$

Then $\operatorname{hit}(X)$ is (equivalent to) a $\Pi_{2}$-formula and

$$
\text { uni-WD }{ }_{\text {hit }} \leq^{\text {serf }} \text { vert-HITTING-SET, }
$$

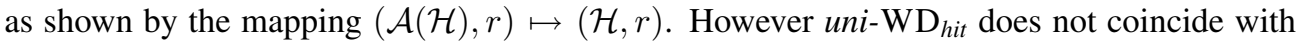
vert-HitTING-SET, because the former has the parameter $r \cdot \log (|V|+|E|)$ and the latter the parameter $r \cdot \log |V|$. Note that $|E|$ can be as large as $2^{|V|}$.

Therefore, we consider a more general reparameterization of the problem Fagin-defined by a formula $\varphi(X)$, namely a relativized version, where a subset of the universe is part of the instance. This subset must contain the solution:

uni-rela- $\mathrm{WD}_{\varphi}$

Instance: $\quad$ A structure $\mathcal{A}$, a set $U \subseteq A$, and $r \in \mathbb{N}$.

Parameter: $\quad r \cdot \log |U|$.

Question: Does there exist $S \subseteq U$ of size $r$ such that $\mathcal{A} \models \varphi(S)$ ?

As shown by the reduction $(\mathcal{A}, r) \mapsto(\mathcal{A}, A, r)$, we have

$$
\text { uni- } \mathrm{WD}_{\varphi} \leq^{\text {serf }} \text { uni-rela- } \mathrm{WD}_{\varphi}
$$

hence, by the Miniaturization Theorem

$$
\mathscr{M}\left(\text { uni- }-\mathrm{WD}_{\varphi}\right) \leq^{\mathrm{fpt}} \mathscr{M}\left(\text { uni-rela }-\mathrm{WD}_{\varphi}\right) .
$$

Furthermore, the reduction $(\mathcal{H}, r) \mapsto(\mathcal{A}(\mathcal{H}), V, r)$ shows that

$$
\text { vert-HITTING-SET } \leq{ }^{\text {serf }} \text { uni-rela-WD } \mathrm{Wit}_{\text {. }} \text {. }
$$

The main technical lemma of this section reads as follows:

Lemma 23. Let $t \geq 1$ and $\varphi(X) \in \Pi_{t}$. Then $\mathscr{M}\left(\right.$ uni-rela- $\left.\mathrm{WD}_{\varphi}\right) \in \mathrm{W}[t]$.

Proof: By Theorem 16 and the Miniaturization Theorem, we only need to prove that there exists some $d \geq 1$ such that

$$
\text { uni-rela- } \mathrm{WD}_{\varphi} \leq^{\text {serf }} \operatorname{var}-\mathrm{WSAT}\left(\Gamma_{t, d}\right) .
$$

We argue as in the proof of [8, Lemma 7.2]: For the sake of simplicity we assume $t=2$ and

$$
\varphi(X)=\forall x \exists y \bigvee_{i \in I} \bigwedge_{j \in J} \lambda_{i j},
$$

where all $\lambda_{i j}$ are literals. We let $d:=\max \{2,|J|\}$. To every structure $\mathcal{A}$ of the vocabulary of $\varphi$ and every $U \subseteq A$ we assign a $\Gamma_{2, d}$-formula $\alpha$ such that for all $r \in \mathbb{N}$ :

$$
(\mathcal{A}, U, r) \in \text { uni-rela- } \mathrm{WD}_{\varphi} \quad \Longleftrightarrow \quad(\alpha, r) \in \operatorname{var}-\mathrm{WSAT}\left(\Gamma_{t, d}\right) .
$$

For every $a \in U$ let $Y_{a}$ be a propositional variable with the intended meaning " $a$ is in the (interpretation of the) relation symbol $X$ ". We define $\alpha^{\prime}$ by

$$
\alpha^{\prime}:=\bigwedge_{a \in A} \bigvee_{\substack{b \in A \\ i \in I}} \gamma_{i, a, b},
$$

where $\gamma_{i, a, b}$ is the conjunction obtained from $\bigwedge_{j \in J} \lambda_{i j}$ as follows: 
- If $a \in U$, then we replace literals $X x$ by $Y_{a}$ and $\neg X x$ by $\neg Y_{a}$; similarly, if $b \in U$, then we replace literals $X y$ by $Y_{b}$ and $\neg X y$ by $\neg Y_{b}$.

- If $a \notin U$, then we omit $\lambda_{i j}=\neg X x$, and we omit the whole $\gamma_{i, a, b}$ if $\lambda_{i, j}=X x$; similarly we proceed for $b \notin U$ with the literals $\neg X y$ and $X y$.

- If $\lambda_{i j}$ does not contain the relation variable $X$, then we omit $\lambda_{i j}$ if $\mathcal{A} \models \lambda_{i j}(a, b)$, and we omit the whole $\gamma_{i, a, b}$ if $\mathcal{A} \not \models \lambda_{i j}(a, b)$. Here $\mathcal{A} \models \lambda_{i j}(a, b)$ means that $\lambda_{i j}$ holds in $\mathcal{A}$ if $x$ and $y$ are interpreted by $a$ and $b$, respectively.

Then for an arbitrary $S \subseteq U$ one easily verifies that

$$
\mathcal{A} \models \varphi(S) \quad \Longleftrightarrow \quad\left\{Y_{a} \mid a \in S\right\} \text { satisfies } \alpha^{\prime} .
$$

We pass to the formula

$$
\alpha:=\alpha^{\prime} \wedge \bigwedge_{a \in U}\left(Y_{a} \vee \neg Y_{a}\right)
$$

to ensure that all variables $Y_{a}$ with $a \in U$ occur in $\alpha$. Then the equivalence (14) holds and as $\alpha$ contains exactly $|U|$ many variables, the mapping

$$
(\mathcal{A}, U, r) \mapsto(\alpha, r)
$$

is a serf-reduction from uni-rela- $\mathrm{WD}_{\varphi}$ to $\operatorname{var}-\mathrm{WSAT}\left(\Gamma_{t, d}\right)$.

Proof of Theorem 22: By Proposition 5

$$
p-\mathrm{WD}_{\varphi} \leq^{\mathrm{fpt}} \mathscr{M}\left(u n i-\mathrm{WD}_{\varphi}\right) .
$$

Then, by Lemma 23 and the $\mathrm{W}[t]$-hardness of $p$ - $\mathrm{WD}_{\varphi}$, we conclude that $\mathscr{M}\left(u n i-\mathrm{WD}_{\varphi}\right)$ is $\mathrm{W}[t]$-complete.

As an application we get:

Proposition 24. The miniaturizations of the following problems are $\mathrm{W}[2]$-complete under fptreductions:

size-HitTING-SET and vert-HitTING-SET,

where

size-HitTING-SET

Instance: A hypergraph $\mathcal{H}=(V, E)$ and $r \geq 1$.

Parameter: $\quad r \cdot \log (|V|+|E|)$.

Question: Does there exist a set $S \subseteq V$ of size $r$ such that $S \cap e \neq \emptyset$

for all $e \in E$ ?

Proof: Clearly, size-HitTING-SET $\equiv^{\text {serf }} u n i-\mathrm{WD}_{h i t}$. Thus, by (10) and (13), we have

$$
\text { size-HITTING-SET } \equiv^{\text {serf }} u n i-\mathrm{WD}_{h i t} \leq^{\text {serf }} \text { vert-HITTING-SET } \leq^{\text {serf }} \text { uni-rela-WD } \mathrm{W}_{\text {hit }} .
$$

Furthermore, we know that the parameterized hitting set problem and thus $p$ - $\mathrm{WD}_{h i t}$ are $\mathrm{W}[2]$ complete. Hence, so is $\mathscr{M}\left(\right.$ uni- $\left.\mathrm{WD}_{\text {hit }}\right)$ by Theorem 22. Therefore $\mathscr{M}\left(\right.$ uni-rela- $\left.\mathrm{WD}_{\text {hit }}\right)$ is $\mathrm{W}[2]$-complete by (12) and Lemma 23. Now we get our claim from (15) by the Miniaturization Theorem.

As an immediate consequence, we get:

Corollary 25. If there is an algorithm solving HITTING-SET in time $2^{0^{\text {eff }}(r \cdot \log (|V|+|E|))}$. $\|\mathcal{H}\|^{O(1)}$, then HITTING-SET is solvable in time $2^{e^{\text {eff }}(r \cdot \log |V|)} \cdot\|\mathcal{H}\|^{O(1)}$. 
We turn to the clique problem. As the W[1]-complete problem $p$-CLIQUE is Fagin-definable by a $\Pi_{1}$-formula, by Theorem 22 we see that $\mathscr{M}$ (uni-CLIQUE) is $\mathrm{W}[1]$-complete. Therefore:

Corollary 26. $\mathrm{W}[1]=$ FPT if and only if one can decide whether a graph $\mathcal{G}=(V, E)$ has a clique of size $k$ in time

$$
2^{o^{\text {eff }}(k \cdot \log |V|)} \cdot|V|^{O(1)} .
$$

Similarly as Theorem 20, one can show (part (2) is again proved by Chen et al. [2]):

Theorem 27. (1) If $\mathrm{W}[1]=$ FPT, then one can decide whether a graph $\mathcal{G}=(V$, E) has a clique of size $k$ in time $|V|^{o^{\text {eff }}(k)}$.

(2) If $\mathrm{M}[1] \neq \mathrm{FPT}$, then one cannot decide whether a graph $\mathcal{G}=(V, E)$ has a clique of size $k$ in time $|V|^{o^{\text {eff }}(k)}$.

We close this section with two open problems. From Proposition 5 we know that

$$
p-\mathrm{WD}_{\varphi} \leq^{\mathrm{fpt}} \mathscr{M}\left(\text { uni- }-\mathrm{WD}_{\varphi}\right)
$$

holds for every formula $\varphi(X)$. Is there a natural Fagin-definable problem $p$ - $\mathrm{WD}_{\varphi}$, for which $\leq^{\mathrm{fpt}}$ cannot be replaced by $\equiv^{\mathrm{fpt}}$ in (16) (modulo complexity theoretic assumptions)? We believe that this could be the case for $p$-CLIQUE-OR-INDEPENDENT-SET, where

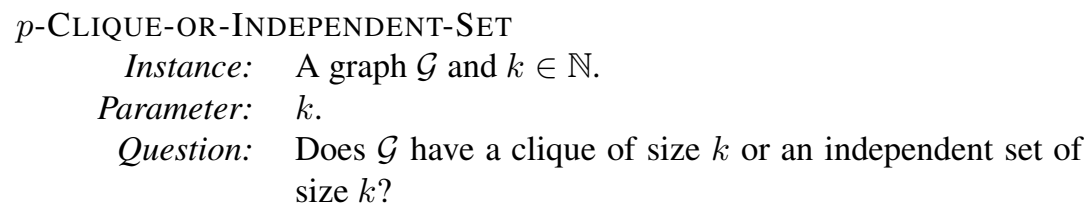

It is known that the problem is fixed-parameter tractable [11]. However, is it solvable in time $2^{o^{\text {eff }}(k \cdot \log |V|)} \cdot|V|^{O(1)}$ ?

By the way, the problem $p$-RED/BLUE-NONBLOCKER (cf. Example 8(c)) can not be written as $p-\mathrm{WD}_{\varphi}$ with a $\Pi_{1}$-formula $\varphi$; it has the form $p-\mathrm{WD}_{\varphi_{0}}$ for some $\Pi_{2}$-formula $\varphi_{0}$. As the problem is $\mathrm{W}[1]$-complete we cannot apply Theorem 22 to get $\mathscr{M}$ (red-RED/BLUE-NoNBLOCKER $) \equiv{ }^{\mathrm{fpt}} p$-RED/BLUE-NONBLOCKER. However we know that this equivalence holds (cf. Proposition 12).

We come to our second open question. We consider the problem of computing the VapnikChervonenkis dimension of a hypergraph. We let

\section{$p$-VC-DIMENSION}

Instance: $\quad$ A hypergraph $\mathcal{H}=(V, E)$ and $r \in \mathbb{N}$.

Parameter: $r$.

Question: $\quad$ Is there is a subset $Y$ of $V$ of size $r$ such for every $Z \subseteq Y$ there is an $e \in E$ such that $Z=Y \cap e$ ?

It is known that $p$-VC-DIMENSION is $\mathrm{W}[1]$-complete. From Proposition 5 we know that

$$
p \text {-VC-DIMENSION } \leq^{\mathrm{fpt}} \mathscr{M}(\text { uni-VC-DIMENSION }) .
$$

Again we do not know whether we can replace $\leq^{\mathrm{fpt}}$ by $\equiv^{\mathrm{fpt}}$. We cannot apply Theorem 10 , as we are not able to prove the corresponding condensation property and we cannot apply Theorem 22, as the problem $p$-VC-DiMENSION is not Fagin-definable by a $\Pi_{1}$-formula. 


\section{Model-checking problems}

It is well-known (see Theorem 28) that the classes of the W-hierarchy can also be characterized in terms of model-checking problems. However, are there model-checking problems complete for the preimages of the classes of the W-hierarchy or, equivalently, are there miniaturizations of model-checking problems complete for the classes of the W-hierarchy? We determine such problems and give some applications.

If $\varphi$ is a first-order formula, we write $\varphi\left(x_{1}, \ldots, x_{m}\right)$ to indicate that the free variables in $\varphi$ are among $x_{1}, \ldots, x_{m}$. The formula $\varphi$ is a sentence if no variable is free in $\varphi$.

The parameterized model-checking problem for a class $\Phi$ of first-order formulas is

$$
\begin{aligned}
p-\mathrm{MC}(\Phi) & \\
\text { Instance: } & \text { A structure } \mathcal{A} \text { and a sentence } \varphi \in \Phi . \\
\text { Parameter: } & |\varphi| . \\
\text { Question: } & \mathcal{A}=\varphi ?
\end{aligned}
$$

We turn to the fragments of first-order logic whose model-checking problems are complete for the classes $\mathrm{W}[t]$. Recall that for $t \geq 0$ and $u \geq 1$ a formula is in $\Pi_{t, u}^{0}$ if it is in $\Pi_{t}$ and all quantifier blocks have length bounded by $u$. For $t, u \geq 1$ a formula is in $\Sigma_{t, u}$ if it has the form

$$
\exists x_{1} \ldots \exists x_{r} \psi
$$

with $r \geq 1$ and $\psi \in \Pi_{t-1, u}^{0}$. Note that $\Sigma_{1}=\Sigma_{1, u}$. Finally, for $s \geq 1$ and a class $\Phi$ of first-order formulas, let $\Phi[s]$ denote the class of formulas in $\Phi$ whose vocabulary has arity $\leq s$.

Theorem 28. Let $t, u \geq 1$ and $s \geq 2$. Then $p-\operatorname{MC}\left(\Sigma_{t, u}\right)$ and $p-\operatorname{MC}\left(\Sigma_{t, u}[s]\right)$ are $\mathrm{W}[t]$ complete under fpt-reductions.

Fix $t, u \geq 1$. The following nondeterministic polynomial time algorithm solves the problem $p-\operatorname{MC}\left(\Sigma_{t, u}\right)$ : Given an instance $(\mathcal{A}, \varphi)$, where $\varphi=\exists x_{1} \ldots \exists x_{r} \psi$ with $\psi \in \Pi_{t-1, u}^{0}$, the algorithm guesses elements $a_{1}, \ldots, a_{r}$ of $A$ (that is, $r \cdot \log |A|$ bits) and then checks if $a_{1}, \ldots, a_{r}$ satisfy $\psi$, that is, if $\mathcal{A}=\psi\left(a_{1}, \ldots, a_{r}\right)$. Note that $\mathcal{A} \models \psi\left(a_{1}, \ldots, a_{r}\right)$ can be checked in time $O\left(|\varphi| \cdot\|\mathcal{A}\|^{t \cdot u}\right)$.

This yields the canonical reparameterization uni-lead-MC $(\Phi)$ of $p-\operatorname{MC}\left(\Sigma_{t, u}\right)$. For a $\Sigma_{t, u^{-}}$ formula $\varphi$ as above we set $\operatorname{lead}(\varphi):=r$; that is, $\operatorname{lead}(\varphi)$ is the number of variables quantified in the leading existential quantifier block.

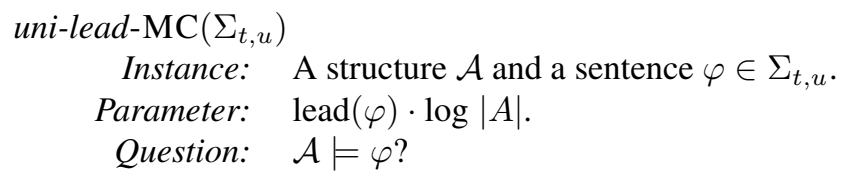

By Theorem 28 one might conjecture that for $s \geq 2$

$$
\begin{gathered}
\mathscr{M}\left(\text { uni-lead-MC }\left(\Sigma_{t, u}\right)\right) \text { and } \mathscr{M}\left(\text { uni-lead-MC }\left(\Sigma_{t, u}\right)[2]\right) \text { are } \mathrm{W}[t] \text {-complete under } \\
\text { fpt-reductions. }
\end{gathered}
$$

However this is unlikely as:

Theorem 29. For $t, u \geq 1$

$$
\mathscr{M}\left(\text { uni-lead-MC }\left(\Sigma_{t, u}\right)\right) \quad \text { and } \mathscr{M}\left(\text { uni-lead-MC }\left(\Sigma_{t, u}\right)[2]\right)
$$

are W[SAT]-complete under fpt-reductions. 
Proof: By Corollary 19 we know that $\mathscr{M}(p$-SAT(PROP $))$ is $\mathrm{W}[\mathrm{SAT}]$-complete. Therefore by the Miniaturization Theorem it suffices to show that

$$
p \text {-SAT }(\mathrm{PROP}) \leq \leq^{\text {serf }} \text { uni-lead-MC }\left(\Sigma_{1}\right)[2] \text { and uni-lead-MC }\left(\Sigma_{t, u}\right) \leq^{\text {serf }} p \text {-SAT(PROP). }
$$

$p$-SAT(PROP) $\leq$ serf uni-lead-MC $\left(\Sigma_{1}\right)[2]:$ Let $\tau$ be the empty vocabulary and $\mathcal{A}$ the $\tau$ structure with $A:=\{0,1\}$. Let $\alpha$ be a propositional formula. For every propositional variable $X$ let $y(X)$ and $z(X)$ be variables of first-order logic. Consider the quantifier-free first-order formula obtained from $\alpha$ by replacing every occurrence of each variable $X$ by $y(X)=z(X)$ and let $\varphi_{\alpha}$ be the $\Sigma_{1}$-sentence obtained from it by existentially quantifying all variables occurring in it. Then $\alpha \mapsto\left(\mathcal{A}, \varphi_{\alpha}\right)$ is the desired reduction.

uni-lead-MC $\left(\Sigma_{t, u}\right) \leq{ }^{\operatorname{serf}} p$-SAT(PROP): Let $(\mathcal{A}, \varphi)$ be an instance of uni-lead-MC $\left(\Sigma_{t, u}\right)$ and $r:=\operatorname{lead}(\varphi)$, say, $\varphi=\exists x_{1} \ldots \exists x_{r} \psi$. We define a propositional formula $\alpha$ with $r \cdot \log |A|$ variables such that

$$
\mathcal{A} \models \varphi \quad \Longleftrightarrow \quad \alpha \text { is satisfiable. }
$$

This will yield the desired serf-reduction. The $r \cdot \log |A|$ variables of $\alpha$ are grouped into $r$ blocks of length $\log |A|$,

$$
\bar{X}_{1}, \ldots, \bar{X}_{r}
$$

with $\bar{X}_{i}:=X_{i, 1}, \ldots, X_{i, \log |A|}$ for $i \in[r]$. We may assume that $A:=\{0, \ldots,|A|-1\}$ and we identify each $a \in A$ with its binary representation of length $\log |A|$.

The formula $\alpha$ will have the property that for every $\bar{a}=\left(a_{1}, \ldots, a_{r}\right) \in A^{r}$

$$
\mathcal{A} \models \psi(\bar{a}) \quad \Longleftrightarrow \quad \text { the assignment } S_{\bar{a}} \text { satisfies } \alpha,
$$

where for each $i \in[r]$ and $j \in[\log |A|]$

$$
X_{i, j} \in S_{\bar{a}} \Longleftrightarrow \quad \text { the } j \text { th bit of } a_{i} \text { is } 1 \text {. }
$$

As a first step to get $\alpha$, for each $e \in A$ and $i \in[r]$ we introduce the propositional formula $\gamma_{e, i}:=\bigwedge_{j \in[\log |A|]} \lambda_{e, i, j}$ with

$$
\lambda_{e, i, j}:= \begin{cases}X_{i, j} & \text { the } j \text { th bit of } e \text { is } 1, \\ \neg X_{i, j} & \text { otherwise. }\end{cases}
$$

Then for every $\bar{a}=\left(a_{1}, \ldots, a_{r}\right) \in A^{r}$

$$
e=a_{i} \quad \Longleftrightarrow \quad \text { the assignment } S_{\bar{a}} \text { satisfies } \gamma_{e, i} \text {. }
$$

and for each assignment $S \subseteq\left\{\bar{X}_{1}, \ldots, \bar{X}_{r}\right\}$ we have

$$
S \text { satisfies } \bigwedge_{i \in[r]} \bigvee_{e \in A} \gamma_{e, i} \quad \Longleftrightarrow \quad \text { there exists some } \bar{a} \in A^{r} \text { such that } S=S_{\bar{a}}
$$

For simplicity, let us assume that $t=2, u=1$ and that $\psi=\forall y \exists z\left(R_{1} x_{1} y x_{3} z \wedge \neg R_{2} y x_{2}\right)$. Then we let

$$
\alpha:=\bigwedge_{i \in[r]} \bigvee_{e \in A} \gamma_{e, i} \wedge \bigwedge_{a \in A} \bigvee_{b \in A}\left(\bigvee_{\begin{array}{c}
e_{1}, e_{3} \in A \text { with } \\
\left(e_{1}, a, e_{3}, b\right) \in R_{1}^{\mathcal{A}}
\end{array}}\left(\gamma_{e_{1}, 1} \wedge \gamma_{e_{3}, 3}\right) \wedge \neg \bigvee_{\substack{e_{2} \in A \text { with } \\
\left(a, e_{2}\right) \in R_{2}^{\mathcal{A}}}} \gamma_{e_{2}, 2}\right)
$$

Using (19) and (18) it is easy to verify (17). As $|\alpha| \leq|A|^{t \cdot u} \cdot|\varphi| \cdot\|\mathcal{A}\|$, the formula $\alpha$ can be computed from $\mathcal{A}$ and $\varphi$ in polynomial time.

To obtain a W[t]-complete problem we have to consider a subclass of $\Sigma_{t, u}$. A $\Sigma_{t, u}^{*}$-formula is a $\Sigma_{t, u}$-formula 
- if $t$ is even and its quantifier-free part is in conjunctive normal form,

- if $t$ is odd and its quantifier-free part is in disjunctive normal form.

Note that $p-\operatorname{MC}\left(\Sigma_{t, u}^{*}\right) \equiv{ }^{\mathrm{fpt}} p-\mathrm{MC}\left(\Sigma_{t, u}\right)$, as in time allowed by an fpt-reduction, the quantifier-free part of a formula $\varphi$ in $\Sigma_{t, u}$ can be transformed into conjunctive or disjunctive normal form (as $|\varphi|$ is the parameter). However, it is not clear whether uni-lead-MC $\left(\Sigma_{t, u}^{*}\right) \equiv^{\text {serf }}$ uni-lead-MC $\left(\Sigma_{t, u}\right)$. It seems improbable, as:

Theorem 30. Let $t, u \geq 1$ and $s \geq 2$. Then $\mathscr{M}\left(\right.$ uni-lead-MC $\left.\left(\Sigma_{t, u}^{*}[s]\right)\right)$ is $\mathrm{W}[t]$-complete under fpt-reductions.

Proof: Fix $t, u \geq 1$ and $s \geq 2$. By Proposition 5 we know that

$$
p-\operatorname{MC}\left(\Sigma_{t, u}^{*}[s]\right) \leq{ }^{\mathrm{fpt}} \mathscr{M}\left(\text { uni-lead-MC }\left(\Sigma_{t, u}^{*}[s]\right)\right)
$$

and thus $\mathscr{M}\left(\right.$ uni-lead-MC$\left.\left(\Sigma_{t, u}^{*}[s]\right)\right)$ is $\mathrm{W}[t]$-hard. Therefore, by Theorem 16 it suffices to show that

$$
\text { uni-lead-MC }\left(\Sigma_{t, u}^{*}[s]\right) \leq^{\text {serf }} \operatorname{var}-\mathrm{WSAT}\left(\Gamma_{t, s}\right) \text {. }
$$

Let $(\mathcal{A}, \varphi)$ be an instance of uni-lead-MC $\left(\Sigma_{t, u}^{*}[s]\right)$ with $r:=\operatorname{lead}(\varphi)$, say, $\varphi=\exists x_{1} \ldots \exists x_{r} \psi$.

We argue as in the proof of [8, Lemma 7.23]. We assume that $t$ is odd (in case $t$ is even one argues similarly) and for notational simplicity that $t=3$. Hence

$$
\psi=\forall \bar{y} \exists \bar{z} \bigvee_{i \in[m]} \bigwedge_{j \in\left[n_{i}\right]} \lambda_{i j}
$$

where $|\bar{y}|,|\bar{z}| \leq u$ and where the $\lambda_{i j}$ are literals.

Let us first assume that $r \leq|A|$. We shall define a propositional formula $\alpha \in \Gamma_{t, s}$ such that

$$
\mathcal{A} \models \varphi \Longleftrightarrow \alpha \text { is } r \text {-satisfiable. }
$$

The formula $\alpha$ will have propositional variables $X_{i, a}$ for all $i \in[r]$ and $a \in A$. The intended meaning of $X_{i, a}$ is: "First-order variable $x_{i}$ takes value $a$." Note that an assignment of weight $r$ satisfies the formula

$$
\chi:=\bigwedge_{i \in[r]} \bigwedge_{\substack{a, b \in A \\ a \neq b}}\left(\neg X_{i, a} \vee \neg X_{i, b}\right)
$$

if and only if for every $i \in[r]$ there is exactly one $a$ such that $X_{i, a}$ is set to TRUE.

We translate the formula in (21) into propositional logic by setting

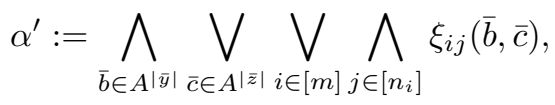

where $\xi_{i j}(\bar{b}, \bar{c})$ is the following formula in $\Delta_{1, s}$ : Let $x_{i_{1}}, \ldots, x_{i_{\ell}}$ be the variables from $x_{1}, \ldots, x_{r}$ in $\lambda_{i j}$; hence, $\ell \leq s$ and $\lambda_{i j}=\lambda_{i j}\left(x_{i_{1}}, \ldots, x_{i_{\ell}}, \bar{y}, \bar{z}\right)$. Then, we set

$$
\xi_{i j}(\bar{b}, \bar{c}):=\bigwedge_{\substack{a_{1}, \ldots, a_{\ell} \in A \\ \mathcal{A} \not \models \lambda_{i j}\left(a_{1}, \ldots, a_{\ell}, \bar{b}, \bar{c}\right)}}\left(\neg X_{i_{1}, a_{1}} \vee \ldots \vee \neg X_{i_{\ell}, a_{\ell}}\right)
$$

Note that $\left(\chi \wedge \alpha^{\prime}\right)$ is $r$-satisfiable if and only if $\mathcal{A} \models \varphi$, and that $\left(\chi \wedge \alpha^{\prime}\right)$ is equivalent to a $\Gamma_{t, s}$-formula $\alpha$ obtainable in time allowed by a serf-reduction; hence, we get (22). As the number of variables of $\alpha$ is $r \cdot|A|$ and, by assumption, $r \leq|A|$, we see that the parameter $r \cdot \log (r \cdot|A|)$ of the instance $(\alpha, r)$ of $\operatorname{var-WSAT}\left(\Gamma_{t, s}\right)$ is $O(r \cdot \log |A|)$ (and $r \cdot \log |A|$ is the parameter of the instance $(\mathcal{A}, \varphi)$ of uni-lead-MC $\left.\left(\Sigma_{t, u}^{*}[s]\right)\right)$.

Now we turn to the case $r>|A|$. One easily sees that we can assume that $|A| \geq 2$ and that $e:=\sqrt{r}$ is a natural number. Clearly, $e \leq|A|^{e}$. Then we introduce a structure $\mathcal{B}$ with universe $A \cup A^{e}$. Its vocabulary contains, among others, a unary relation symbol $U$ with

$$
U^{\mathcal{B}}:=A
$$


Recall that $\psi=\psi\left(x_{1}, \ldots, x_{r}\right)$. Let $u_{1}, \ldots, u_{e}$ be new first-order variables. We intend to interpret them in $\mathcal{B}$ by elements from $A^{e}$, more precisely, $u_{1}$ represents the (interpretation of the) tuple $\left(x_{1}, \ldots, x_{e}\right)$, the variable $u_{2}$ the tuple $\left(x_{e+1}, \ldots, x_{2 e}\right), \ldots$ For every atomic subformula $\lambda$ of $\varphi$, the vocabulary contains a relation symbol $R(\lambda)$. For example, if $\lambda=$ $R x_{2} y_{3} z_{4} x_{9} z_{1}$ and $e=4$, then the relation symbol $R(\lambda)$ will be 5 -ary and

$$
\left(\bar{a}, \bar{b}, c, d, d^{\prime}\right) \in R(\lambda)^{\mathcal{B}} \Longleftrightarrow\left(\bar{a}, \bar{b} \in A^{e}, c, d, d^{\prime} \in A \text {, and }\left(a_{2}, c, d, b_{1}, d^{\prime}\right) \in R^{\mathcal{A}}\right),
$$

where $a_{2}$ is the second member of $\bar{a}$ and $b_{1}$ the first member of $\bar{b}$.

We set

$$
\varphi^{\prime}:=\exists u_{1} \ldots \exists u_{e} \forall \bar{y} \exists \bar{z}\left(\bigwedge_{i \in[e]} \neg U e_{i} \wedge\left(\bigwedge_{i \in[|\bar{y}|]} U y_{i} \rightarrow\left(\bigwedge_{i \in[|\bar{z}|]} U z_{i} \wedge \bigvee_{i \in[m]} \bigwedge_{j \in\left[n_{i}\right]} \lambda_{i j}^{\prime}\right)\right),\right.
$$

where $\lambda_{i j}^{\prime}$ is $R\left(\lambda_{i j}\right) u_{1} u_{3} y_{3} z_{4} z_{1}$ in case $\lambda_{i j}=R x_{2} y_{3} z_{4} x_{9} z_{1}$. In time polymomial in $\varphi^{\prime}$ we obtain an equivalent formula $\varphi^{\prime \prime}$ in $\Sigma_{t, u}^{*}$. Therefore the transition $(\mathcal{A}, \varphi) \mapsto\left(\mathcal{B}, \varphi^{\prime \prime}\right)$ can be carried out in time polynomial in $|A|^{e}$ and

$$
|A|^{e}=|A|^{\sqrt{r}}=2^{\sqrt{r} \cdot \log |A|}=2^{o^{\text {eff }}(r \cdot \log |A|)},
$$

the last equality holding as $r>|A|$. Furthermore, note that

$$
e \cdot \log \left|A \cup A^{e}\right| \in O(r \cdot \log |A|) .
$$

For $i \in[e]$ and $\bar{a} \in A^{e}$ we let $X_{i, \bar{a}}$ be a propositional variable. Now we transform the formula $\varphi^{\prime \prime}$ into a propositional formula $\beta$ essentially as we did it previously for the formula $\varphi$. Note that lead $\left(\varphi^{\prime \prime}\right)=e \leq|A|^{e} \leq|B|$. Altogether, it is not hard to verify using (24) and (25) that we obtain the desired serf-reduction.

We give an application of the preceding result. Let $s \geq 1$. The parameterized homomorphism problem $p-\mathrm{HOM}[s]$ for structures of arity $\leq s$ is the following problem:

$$
\begin{aligned}
p-\mathrm{Hom}[s] & \\
\text { Instance: } & \text { Structures } \mathcal{A} \text { and } \mathcal{B} \text { of arity } \leq s . \\
\text { Parameter: } & \|\mathcal{A}\| . \\
\text { Question: } & \text { Is there a homomorphism from } \mathcal{A} \text { to } \mathcal{B} ?
\end{aligned}
$$

The following is known (cf. [8]).

Theorem 31. Let $s \geq 2$. Then $p-\mathrm{HoM}[s]$ is $\mathrm{W}[1]$-complete under fpt-reductions.

The canonical reparameterization of $p-\mathrm{HOM}[s]$ is the problem

$$
\begin{aligned}
\text { uni-Hom }[s] & \\
\text { Instance: } & \text { Structures } \mathcal{A} \text { and } \mathcal{B} \text { of arity } \leq s . \\
\text { Parameter: } & |A| \cdot \log |B| . \\
\text { Question: } & \text { Is there a homomorphism from } \mathcal{A} \text { to } \mathcal{B} ?
\end{aligned}
$$

A further reparameterization of $p-\mathrm{HOM}[s]$ is

$$
\begin{aligned}
\text { size-Hom }[s] & \\
\text { Instance: } & \text { Structures } \mathcal{A} \text { and } \mathcal{B} \text { of arity } \leq s . \\
\text { Parameter: } & \|\mathcal{A}\| \cdot \log \|\mathcal{B}\| . \\
\text { Question: } & \text { Is there a homomorphism from } \mathcal{A} \text { to } \mathcal{B} ?
\end{aligned}
$$

By Proposition 5 we have: 
Lemma 32. Let $s \geq 1$. Then

$$
p-\mathrm{HOM}[s] \leq^{\mathrm{fpt}} \mathscr{M}(\text { size-Hом }[s]) \leq{ }^{\mathrm{fpt}} \mathscr{M}(\text { uni-Ном }[s]) .
$$

We show that the three problems are equivalent:

Theorem 33. Let $s \geq 2$. Then

$$
p-\mathrm{HOM}[s] \equiv^{\mathrm{fpt}} \mathscr{M}(\text { size-Hoм }[s]) \equiv{ }^{\mathrm{fpt}} \mathscr{M}(\text { uni-Ном }[s]) .
$$

Proof: Let $s \geq 2$. By Theorem 31 and (26), it suffices to show that $\mathscr{M}($ uni-Hom $[s]) \in \mathrm{W}[1]$. For that purpose, we prove

$$
\text { uni-HOM }[s] \leq^{\text {serf }} \text { uni-lead-MC }\left(\Sigma_{1,1}^{*}[s]\right) .
$$

Then the result follows from Theorem 30 and the Miniaturization Theorem.

Let $(\mathcal{A}, \mathcal{B})$ be an instance of uni-HoM $[s]$. Clearly we can assume that both structures have the same vocabulary $\tau$ (otherwise, $(\mathcal{A}, \mathcal{B})$ is a negative instance of uni-HOM $[s])$. Let $k:=|A|$ and $A=\left\{a_{1}, \ldots, a_{k}\right\}$. Then we let

$$
\varphi:=\exists x_{1} \ldots \exists x_{k} \bigwedge_{r \in[s]} \bigwedge_{\substack{R \in \tau \\ \text { of arity } r}} \bigwedge_{\left(a_{i_{1}}, \ldots, a_{i_{r}}\right) \in R^{\mathcal{A}}} R x_{i_{1}} \ldots x_{i_{r}} .
$$

It is easy to see that

$$
\text { there is a homomorphism from } \mathcal{A} \text { to } \mathcal{B} \quad \Longleftrightarrow \quad \mathcal{B}=\varphi \text {. }
$$

This gives the desired serf-reduction from uni-HoM $[s]$ to uni-lead-MC $\left(\sum_{1,1}^{*}[s]\right)$.

Corollary 34. Let $s \geq 2$. $\mathscr{M}($ size- $\mathrm{Hom}[s])$ and $\mathscr{M}($ uni-Hom $[s])$ are $\mathrm{W}[1]$-complete under fpt-reductions.

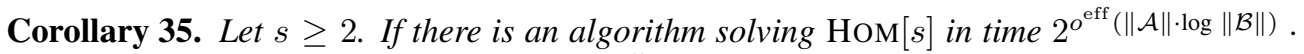
$\|\mathcal{B}\|^{O(1)}$, then $\mathrm{HOM}[s]$ is solvable in time $2^{\alpha^{\text {eff }}(|A| \cdot \log |B|)} \cdot\|\mathcal{B}\|^{O(1)}$.

\section{The preimage of the A-hierarchy}

In this section, among others, we determine model-checking problems complete for the preimages of the classes of the A-hierarchy.

The A-hierarchy can be characterized in terms of model-checking problem (see [8]):

Proposition 36. For $t \geq 1$ and $s \geq 2$ the problems $p-\mathrm{MC}\left(\Sigma_{t}\right)$ and $p-\mathrm{MC}\left(\Sigma_{t}[s]\right)$ are $\mathrm{A}[\mathrm{t}]-$ complete under fpt-reductions.

An example of a graph problem complete for A[2] is

$$
\begin{aligned}
& p \text {-CLIQUe-Dominating-SET } \\
& \text { Instance: } \text { A graph } \mathcal{G} \text { and } k, \ell \in \mathbb{N} . \\
& \text { Parameter: } k+\ell . \\
& \text { Question: } \begin{array}{l}
\text { Does } \mathcal{G} \text { contain a set of } k \text { vertices that dom- } \\
\text { inates every clique of size } \ell ?
\end{array}
\end{aligned}
$$

A set $X$ of vertices of $\mathcal{G}=(V, E)$ dominates the set of vertices $Y$ if there are $v \in X$ and $w \in Y$ such that $v=w$ or $\{v, w\} \in E$.

For these problems we have no notion of canonical reparameterization, as their underlying classical problems are not in NP apparently. In fact, it is well-known that the classical problem underlying $p-\operatorname{MC}\left(\Sigma_{t}[s]\right)$ is $\Sigma_{t}^{p}$-complete.

Nevertheless we introduce the following reparameterization of the graph problem 
relations $R(\lambda)^{\mathcal{A}^{*}}$ are defined accordingly (compare the proof of Theorem 30). Then $(\mathcal{A} \models$ $\left.\varphi \Longleftrightarrow \mathcal{A}^{*} \models \varphi^{*}\right)$. As $\left|A^{r}\right|=|A|^{d / k} \leq|A|^{|\operatorname{var}(\varphi)| / k} \cdot|A|$ and $\left|\operatorname{var}\left(\varphi^{*}\right)\right| \leq k \cdot t$, we see that $\operatorname{var}-\operatorname{MC}\left(\Sigma_{t}[s]\right)$ has the $h$-condensation property (where as function $f: \mathbb{N} \rightarrow \mathbb{N}$ according to Definition 7 we take the function $f(k):=k \cdot t)$.

Proof of Proposition 37: In view of the preceding lemma the claim is obvious by Theorem 10.

For $s \geq 2$ the problem $\mathscr{M}\left(\right.$ uni-var- $\left.\mathrm{MC}\left(\Sigma_{t}[s]\right)\right)$ is W[SAT]-hard and therefore it is highly improbable that it is $\mathrm{A}[t]$-complete. As for the W-hierarchy, in order to get a complete problem we have to restrict the model-checking problem to a subclass $\Sigma_{t}^{*}$ of $\Sigma_{t}$ defined as there: A $\Sigma_{t^{-}}$ formula is in $\Sigma_{t}^{*}$

- if $t$ is even and its quantifier-free part is in conjunctive normal form, and

- if $t$ is odd and its quantifier-free part is in disjunctive normal form.

We show:

Theorem 39. Let $t \geq 1$. Then $\mathscr{M}$ (uni-var- $\left.\mathrm{MC}\left(\Sigma_{t}^{*}[2]\right)\right)$ is $\mathrm{A}[t]$-complete under fpt-reductions.

To prove this theorem we need a long detour through propositional logic. We consider the following parameterized problem for a set of propositional formulas $\Gamma$ :

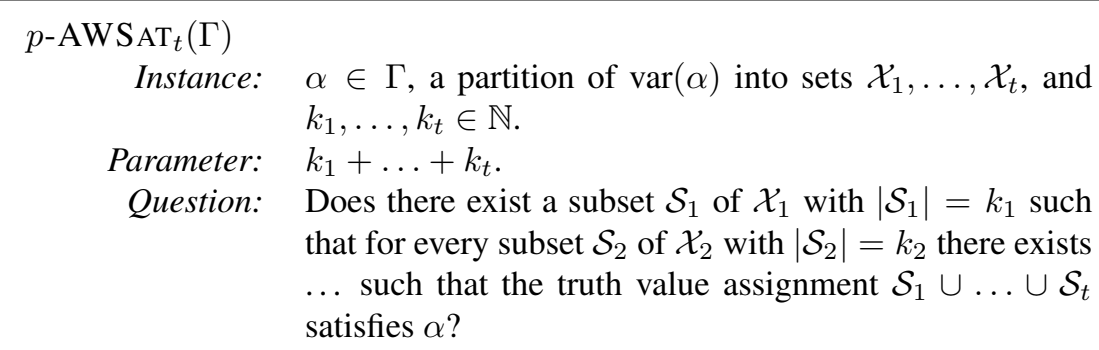

and its reparameterization

$\operatorname{var}-\mathrm{AWSAT}_{t}(\Gamma)$

Instance: $\alpha \in \Gamma$, a partition of the propositional variables of $\alpha$ into sets $\mathcal{X}_{1}, \ldots, \mathcal{X}_{t}$, and $k_{1}, \ldots, k_{t} \in \mathbb{N}$.

Parameter: $\quad\left(k_{1}+\ldots+k_{t}\right) \cdot \log |\operatorname{var}(\alpha)|$.

Question: Does there exist a subset $\mathcal{S}_{1}$ of $\mathcal{X}_{1}$ with $\left|\mathcal{S}_{1}\right|=k_{1}$ such that for every subset $\mathcal{S}_{2}$ of $\mathcal{X}_{2}$ with $\left|\mathcal{S}_{2}\right|=k_{2}$ there exists $\ldots$ such that the truth value assignment $\mathcal{S}_{1} \cup \ldots \cup \mathcal{S}_{t}$ satisfies $\alpha$

It is known [7]:

Proposition 40. Ift $\geq 1$ is even, then $p$ - $\mathrm{AWSAT}_{t}\left(\Delta_{1,2}^{+}\right)$is $\mathrm{A}[t]$-complete under fpt-reductions, and if $t \geq 1$ is odd, then so is $p$ - $\mathrm{AWSAT}_{t}\left(\Gamma_{1,2}^{-}\right)$.

We first prove (thereby already getting a problem complete in the preimage of $\mathrm{A}[t]$ ):

Proposition 41. If $t \geq 1$ is even, then $\mathscr{M}\left(\right.$ var- $\left.\mathrm{AWSAT}_{t}\left(\Delta_{1,2}^{+}\right)\right)$is $\mathrm{A}[t]$-complete under fptreductions, and if $t \geq 1$ is odd, then so is $\mathscr{M}$ (var- $\left.\mathrm{AWSAT}_{t}\left(\Gamma_{1,2}^{-}\right)\right)$.

Sketch of a proof: For simplicity we consider the case $t=2$ : Then it suffices to show $p$ - $\operatorname{AWSAT}_{2}\left(\Delta_{1,2}^{+}\right) \leq{ }^{\mathrm{fpt}} \mathscr{M}\left(\operatorname{var}-\mathrm{AWSAT}_{2}\left(\Delta_{1,2}^{+}\right)\right) \leq{ }^{\mathrm{fpt}} p-\mathrm{MC}\left(\Sigma_{2}[2]\right)$.

$p$ - $\operatorname{AWSAT}_{2}\left(\Delta_{1,2}^{+}\right) \leq \leq^{\mathrm{fpt}} \mathscr{M}\left(\operatorname{var}-\mathrm{AWSAT}_{2}\left(\Delta_{1,2}^{+}\right)\right)$: This is clear by Proposition 5.

$\mathscr{M}\left(\right.$ var-AWS $\left.\operatorname{AWT}_{2}\left(\Delta_{1,2}^{+}\right)\right) \leq^{\mathrm{fpt}} p-\mathrm{MC}\left(\Sigma_{2}[2]\right)$ : Let $\left(\alpha, \mathcal{X}_{1}, \mathcal{X}_{2}, k_{1}, k_{2}, m\right)$ be an instance of $\mathscr{M}\left(\right.$ var- $\left.\mathrm{AWSAT}_{2}\left(\Delta_{1,2}^{+}\right)\right)$. Let $k:=k_{1}+k_{2}$. The parameter of this instance is

$$
\ell:=\left\lceil\frac{k \cdot \log |\operatorname{var}(\alpha)|}{\log m}\right\rceil .
$$


The case $\ell>k$ is easy, so let us assume that $\ell \leq k$. Note that

$$
|\operatorname{var}(\alpha)|^{k / \ell} \leq m .
$$

From the proof of $[8$, Lemma 8.18] we know that

$$
\left(\alpha, \mathcal{X}_{1}, \mathcal{X}_{2}, k_{1}, k_{2}\right) \in p \text {-AWSAT } 2\left(\Delta_{1,2}^{+}\right) \Longleftrightarrow \mathcal{A} \models \varphi_{k_{1}, k_{2}}
$$

with

$$
\begin{aligned}
\varphi_{k_{1}, k_{2}}:=\exists x_{1} \ldots \exists x_{k_{1}} & \left(\bigwedge_{i \in\left[k_{1}\right]} P_{1} x_{i} \wedge \bigwedge_{1 \leq i<j \leq k_{1}} x_{i} \neq x_{j} \wedge \forall x_{k_{1}+1} \ldots \forall x_{k_{1}+k_{2}}\right. \\
& \left.\left(\left(\bigwedge_{k_{1}+1 \leq i \leq k_{1}+k_{2}} P_{2} x_{i} \wedge \bigwedge_{k_{1}+1 \leq i<j \leq k_{1}+k_{2}} x_{i} \neq x_{j}\right) \rightarrow \bigvee_{1 \leq i, j \leq k} R x_{i} x_{j}\right)\right),
\end{aligned}
$$

for unary relation symbols $P_{1}, P_{2}$ and a binary $R$; moreover $|A|=\operatorname{var}(\alpha)$ and

$$
R^{\mathcal{A}}:=\left\{(i, j) \mid\left(X_{i} \wedge X_{j}\right) \text { is a term of } \alpha\right\}
$$

(we identify terms of the form $X$ by $(X \wedge X)$ ).

Note that to get an fpt-reduction to $p-\operatorname{MC}\left(\Sigma_{2}[2]\right)$ we need a formula whose total length (not only the length of the prefix) is bounded in terms of $\ell$. The crucial observation is that due to the homogeneous form of $\varphi_{k_{1}, k_{2}}$ and $R^{\mathcal{A}}$ this formula can be condensed completely, that is, we can condense its prefix and its quantifier-free part. We leave the details to the reader. In this way one gets in time polynomial in $m$ a structure $\mathcal{A}^{*}$ and a $\Sigma_{2}^{*}[2]$-sentence $\varphi^{*}$ of length bounded in terms of $\ell$ such that

$$
\left(\alpha, \mathcal{X}_{1}, \mathcal{X}_{2}, k_{1}, k_{2}\right) \in p-\operatorname{AWSAT}_{2}\left(\Delta_{1,2}^{+}\right) \Longleftrightarrow \mathcal{A}^{*} \models \varphi^{*} .
$$

Sketch of a proof of Theorem 39: $p-\operatorname{MC}\left(\Sigma_{t}[2]\right) \leq{ }^{\mathrm{fpt}} \mathscr{M}\left(\right.$ uni-var- $\left.\mathrm{MC}\left(\Sigma_{t}^{*}[2]\right)\right)$ : As for the given parameterization every sentence in $\Sigma_{t}[2]$ can be transformed into an equivalent sentence in $\Sigma_{t}^{*}[2]$ in fpt-time, we get the result from Proposition 5.

$\mathscr{M}\left(\right.$ uni-var-MC $\left.\left(\Sigma_{t}^{*}[2]\right)\right) \leq{ }^{\mathrm{fpt}} p$ - $\mathrm{MC}\left(\Sigma_{t}[2]\right)$ : For notational simplicity let $t=2$. By the Miniaturization Theorem and Proposition 41 it suffices to show that uni-var-MC $\left(\Sigma_{2}^{*}[s]\right) \leq^{\text {serf }}$ $\operatorname{var}-\mathrm{AWSAT}_{2}\left(\Delta_{1,2}^{+}\right)$.

Let $(\mathcal{A}, \varphi)$ be an instance of uni-var-MC $\left(\Sigma_{2}^{*}[s]\right)$. By standard techniques (cf. [8, Section 8.2]) we can further assume that

$$
\varphi=\exists x_{1} \ldots \exists x_{\ell} \forall y_{1} \ldots \forall y_{m} \bigvee_{i \in[s]} \lambda_{i}
$$

where the $\lambda_{i}$ 's are literals. Let us first assume that $\ell+m \leq \log |A|$.

Now we follow the proof of [8, Lemma 8.18]. We define a formula $\alpha^{\prime}$ with variables

$$
\mathcal{X}:=\left\{X_{i, a} \mid i \in[\ell], a \in A\right\} \quad \text { and } \quad \mathcal{Y}:=\left\{Y_{i, a} \mid i \in[m], a \in A\right\}
$$

such that

$$
\mathcal{A} \models \varphi \quad \Longleftrightarrow \quad\left(\alpha^{\prime}, \mathcal{X}, \mathcal{Y}, h, k\right) \in \mathrm{AWSAT}_{2}(\mathrm{PROP}) .
$$

Then the parameter of the instance on the right hand side is $\leq(\ell+m) \cdot \log (\ell \cdot|A|+m \cdot|A|)$. As $\ell+m \leq \log |A|$, this is bounded in terms of $(\ell+m) \cdot \log |A|$, the parameter of the instance $(\mathcal{A}, \varphi)$ of uni-var-MC $\left(\Sigma_{2}^{*}[s]\right)$. We can set

$$
\alpha^{\prime}:=\gamma_{\mathcal{X}} \wedge\left(\neg \gamma_{\mathcal{Y}} \vee \beta\right)
$$


where

$$
\gamma_{\mathcal{X}}:=\bigwedge_{i \in[\ell]} \bigwedge_{\substack{a, b \in A \\ a \neq b}}\left(\neg X_{i, a} \vee \neg X_{i, b}\right), \quad \gamma_{\mathcal{Y}}:=\bigwedge_{i \in[m]} \bigwedge_{\substack{a, b \in A \\ a \neq b}}\left(\neg Y_{i, a} \vee \neg Y_{i, b}\right),
$$

and

$$
\beta:=\bigvee_{i \in[s]} \bigvee_{\substack{a, b \in A \\ \mathcal{A} \models \lambda_{i}(a, b)}} \delta_{i, a, b}
$$

Here, $\delta_{i, a, b}$ is the following formula: if $\lambda_{i}$ contains variables $x_{3}, y_{2}$, then $\delta_{i, a, b}:=\left(X_{3, a} \wedge\right.$ $\left.Y_{2, b}\right)$; if $\lambda_{i}$ only contains $x_{3}$, then $\delta_{i, a, b}:=\left(X_{3, a} \wedge X_{3, a}\right)$. Note that $(\neg \gamma \mathcal{Y} \vee \beta) \in \Delta_{1,2}^{+}$. So one has to transform $\gamma_{\mathcal{X}} \wedge \ldots$ into an equivalent (in the given context) formula in $\Delta_{1,2}^{+}$. This can be done as in the proof of [8, Lemma 8.18].

The case $\ell+m>\log |A|$ is reduced to preceding one in a similar way as it was done in the proof of Theorem 30 .

As an application we get:

Proposition 42. $\mathscr{M}$ (uni-Clique-Dominating-Set) is A[2]-complete under fpt-reductions.

Proof: Proposition 5 shows that

$$
p \text {-Clique-Dominating-Set } \leq{ }^{\mathrm{fpt}} \mathscr{M}(\text { uni-Clique-Dominating-Set); }
$$

therefore, $\mathscr{M}$ (uni-Clique-Dominating-Set) is A[2]-hard under fpt-reductions. To obtain membership in $\mathrm{A}[2]$, by the previous theorem it suffices to show that

$$
\text { uni-CLIQUe-DominAting-SeT } \leq^{\text {serf }} \text { uni-var-MC }\left(\Sigma_{2}^{*}[2]\right) \text {. }
$$

The mapping $(\mathcal{G}, k, \ell) \mapsto\left(\mathcal{G}, \varphi_{k, \ell}\right)$ is a such a serf-reduction, where

$$
\begin{aligned}
\varphi_{k, \ell}:=\exists x_{1} \ldots \exists x_{k} \forall y_{1} \ldots \forall y_{\ell} & \left(\bigwedge_{1 \leq i<j \leq k} x_{i} \neq x_{j}\right. \\
& \left.\wedge\left(\bigvee_{1 \leq i<j \leq \ell} \neg E y_{i} y_{j} \vee \bigvee_{i \in[k], j \in[\ell]}\left(x_{i}=y_{j} \vee E x_{i} y_{j}\right)\right)\right) .
\end{aligned}
$$

\section{Remarks and conclusions}

We introduced the notion of canonical (re)parameterization of a (parameterized) problem and showed, among others, that this reparameterization is the preimage under the miniaturization mapping of Fagin-definable problems complete for some level of the W-hierarchy. One of the main open questions is whether this is true for all Fagin-definable problems.

We have seen that for many natural parameterized problem $(Q, \kappa)$ whose instances are of the form $(x, y)$ with $\kappa(x, y)=|y|$

$$
(Q, \kappa) \text { is fixed-parameter tractable }
$$

$$
\Longleftrightarrow Q \text { is decidable in time } 2^{o^{\text {eff }}(|y| \cdot \log |x|)} \cdot|x|^{O(1)} .
$$

This equivalence is important; for example, it tells us that to prove that the dominating set problem is not solvable in subexponential time is as hard as to prove $\mathrm{W}[2] \neq \mathrm{FPT}$.

We should mention that the direction from right to left in (28) is true for all parameterized problems $(Q, \kappa)$ :

Proposition 43. Let $(Q, \kappa)$ be a parameterized problem over the alphabet $\Sigma$. If there is an algorithm that for every $x \in \Sigma^{*}$ decides whether $x \in Q$ in time $2^{o^{\text {eff }}(\kappa(x) \cdot \log |x|)} \cdot|x|^{O(1)}$, then $(Q, \kappa)$ is fixed-parameter tractable. 
Proof: Let $x \in Q$ be solvable in time $2^{(k \cdot \log |x|) / \iota(k \cdot \log |x|)} \cdot|x|^{O(1)}$, where $k:=\kappa(x)$ and $\iota$ is a nondecreasing and unbounded computable function (thereby we assume that $k \cdot \log |x|$ is sufficiently large).

We define $h: \mathbb{N} \rightarrow \mathbb{N}$ by

$$
h(\ell):=\max \{m \mid \iota(m) \leq \ell\}
$$

(and set $h(\ell):=0$ in case $\{m \mid \iota(m) \leq \ell\}=\emptyset$ ). If $k \cdot \log |x|>h(k)$, then $\iota(k \cdot \log |x|)>k$ and hence

$$
2^{(k \cdot \log |x|) / \iota(k \cdot \log |x|)} \leq|x| .
$$

If $k \cdot \log |x| \leq h(k)$, then

$$
2^{(k \cdot \log |x|) / \iota(k \cdot \log |x|)} \leq 2^{k \cdot \log |x|} \leq 2^{h(k)} .
$$

Hence, for all $x$ we have $2^{(k \cdot \log |x|) / \iota(k \cdot \log |x|)} \leq|x|+2^{h(k)}$.

However to establish the converse direction of (28), we mostly needed the condensation property for each individual problem. There are problems that (apparently) do not have the condensation property, for example $p$-WSAT $\left(\Gamma_{t, d}\right)$. Nevertheless, the equivalence (28) still holds for $p$-WSAT $\left(\Gamma_{t, d}\right)$ by Theorem 16 and the Miniaturization Theorem. The purpose of this section is to give natural problems for which (28) does not hold.

Recall the parameterized model-checking problem for monadic second-order logic (MSO) on trees

$$
\begin{aligned}
p \text {-MC(TREE, MSO) } & \\
\text { Instance: } & \text { A tree } \mathcal{T} \text { and an MSO-sentence } \varphi . \\
\text { Parameter: } & |\varphi| . \\
\text { Question: } & \mathcal{T}=\varphi ?
\end{aligned}
$$

We do not give the precise definition of MSO and of trees, as we do not need them. The reader is referred to [10] or [8, Chapter 10] for the definitions and for details. What we need is contained in Lemma 45.

The equivalence (28) does not hold for $p$-MC(TREE, MSO) (unless FPT $=$ M[1]). In fact, it is well-known that $p$-MC(TREE, MSO) is fixed-parameter tractable [4, 13]. However we show that:

Theorem 44. The miniaturization of the problem

$$
\begin{aligned}
\text { Instance: } & \text { A tree } \mathcal{T} \text { and an MSO-sentence } \varphi . \\
\text { Parameter: } & |\varphi| \cdot \log \|\mathcal{T}\| . \\
\text { Problem: } & \mathcal{T}=\varphi ?
\end{aligned}
$$

is $\mathrm{M}[1]$-hard under serf-reductions.

We need the following lemma which is a very weak version of a result due to Frick and Grohe [10] (see also Corollary 10.27 in [8]). Thereby we use the encoding, computable in polynomial time, of CNF-formulas $\alpha$ by trees $\mathcal{T}(\alpha)$ of size $|\alpha|^{\Theta(1)}$ from [8].

Lemma 45. Let $n \geq 1$ and $h:=\sqrt{n}$. There is a sentence $\varphi_{h}$ of monadic second-order logic, computable in time $O(h)$, such that for all CNF-formulas $\alpha$ with at most $n$ variables we have

$$
\mathcal{T}(\alpha) \models \varphi_{h} \quad \Longleftrightarrow \quad \alpha \text { is satisfiable. }
$$

Proof of Theorem 44: We give a serf-reduction from $p$-SAT(3-CNF), a problem whose miniaturization is known to be M[1]-complete. Let $\alpha$ be a formula in 3-CNF with $n$ variables and let $h:=\sqrt{n}$. By Lemma 45, there is an MSO-sentence $\varphi_{h}$ of length $O(h)$ such that

$$
\alpha \text { is satisfiable } \Longleftrightarrow \mathcal{T}(\alpha) \models \varphi_{h} \text {. }
$$


We know that $\varphi_{h}$ is computable in time $O(h)$ and hence in time polynomial in $|\alpha|$. Moreover

$$
\begin{aligned}
& \left|\varphi_{h}\right| \cdot \log \|\mathcal{T}(\alpha)\| \\
= & O(h \cdot \log |\alpha|) \\
= & O(\sqrt{n} \cdot \log n) \quad \text { (by }|\alpha|=O\left(n^{3}\right), \text { since } \alpha \text { is in 3-CNF) } \\
= & O(n) .
\end{aligned}
$$

Therefore the mapping $\alpha \mapsto\left(\mathcal{T}(\alpha), \varphi_{h}\right)$ is a serf-reduction.

The reader might wonder why we do not use Theorem 29 and its proof to show Theorem 44. In that proof we considered a single fixed structure, which can be easily turned into a tree. The parameter of an instance $(\mathcal{A}, \varphi)$ was lead $(\varphi) \cdot \log |A|$. However lead $(\varphi)=o^{\text {eff }}(|\varphi|)$. Hence checking whether $\mathcal{A} \models \varphi$ can be done in time

$$
|A|^{\operatorname{lead}(\varphi)} \cdot|\varphi| \cdot\|\mathcal{A}\|=2^{o^{\text {eff }}(|\varphi| \cdot \log \|\mathcal{A}\|)} .
$$

\section{References}

[1] K.A. Abrahamson, R.G. Downey, and M.R. Fellows. Fixed-parameter tractability and completeness IV: On completeness for W[P] and PSPACE analogs. Annals of Pure and Applied Logic, 73:235-276, 1995.

[2] J. Chen, B. Chor, M. Fellows, X. Huang, D. Juedes, I. Kanj, and Ge Xia. Tight lower bounds for certain parameterized NP-hard problems. In Proceedings of The 19th IEEE Annual Conference on Computational Complexity (CCC'04), 150-160, 2004.

[3] Y. Chen and M. Grohe. An isomorphism between subexponential and parameterized complexity theory. SIAM Journal on Computing, 37(4):1228-1258, 2007.

[4] J. Doner. Tree acceptors and some of their applications. Journal of Computer and System Sciences, 4:406-451, 1970.

[5] R.G. Downey and M.R. Fellows. Parameterized Complexity. Springer, 1999.

[6] J. Flum and M. Grohe. Parameterized complexity and subexponential time. In the Complexity Column of the Bulletin of the European Association for Theoretical Computer Science (EATCS), 84, October, 2004.

[7] J. Flum and M. Grohe. Model-checking problems as a basis for parameterized intractability. Logical Methods in Computer Science, 1(1), 2005.

[8] J. Flum and M. Grohe. Parameterized Complexity Theory. Springer, 2006.

[9] J. Flum, M. Grohe, and M. Weyer. Bounded fixed-parameter tractability and $\log { }^{2} n$ nondeterministic bits. Journal of Computer and System Sciences, 72:34-71, 2006.

[10] M. Frick and M. Grohe. The complexity of first-order and monadic second-order logic revisited. Annals of Pure and Applied Logic, 130:3-31, 2004.

[11] S. Khot and V. Raman. Parameterized complexity of finding subgraphs with hereditary properties. Theoretical Computer Science, 289(2):997-1008, 2002.

[12] C.H. Papadimitriou and M. Yannakakis. On the complexity of database queries. Journal of Computer and System Sciences, 58:407-427, 1999.

[13] J.W. Thatcher and J.B. Wright. Generalised finite automata theory with an application to a decision problem of second-order logic. Mathematical Systems Theory, 2:57-81, 1968. 